\title{
PROKOPS ANECDOTA UND JUSTINIANS NACHFOLGE
}

\section{Datierung der Anecdota}

Die Gelehrten sind sich bis jetzt nicht über den eigentlichen Zweck von Prokops Anecdota einig. Ein Grund dafür war bis vor kurzem die umstrittene Datierung des Werkes. Prokop selbst sagt viermal in den Anecdota, daß er im 32. Jahr der Regierung Justinians schreibt ${ }^{1}$, eine Aussage, mit der prinzipiell das Jahr 558-59 gemeint sein müßte, da die offizielle Regierung Justinians erst 527 beginnt $^{2}$. Seit Haury ${ }^{3}$ überwiegt aber die Meinung, daß das 32. Jahr eigentlich dem Jahre 550 entspricht, da die tatsächliche Regierung Justinians bereits mit der Krönung seines Onkels Justin 518 beginnt $^{4}$. Diese frühere Datierung ist zwar heute praktisch zur communis opinio geworden ${ }^{5}$, besonders seitdem Geoffrey Greatrex vor einigen Jahren mit überzeugenden Argumenten für das Jahr 550 plädierte ${ }^{6}$. Dennoch wird die spätere Datierung in das Jahr 558 immer noch von verschiedenen Gelehrten vertreten ${ }^{7}$. Das Prooimion der Anecdota wirft auch

${ }^{1}$ An. XVIII.33, XXIII.1 und XXIV.29, 33.

2 F. Dahn, Procopius von Cäsarea. Berlin 1865, 38-39 und W. Teuffel, Procopius, Studien und Charakteristiken zur griechischen und römischen sowie deutschen Literaturgeschichte 1 (1871) 216-217.

3 J. Haury, Procopiana I. Augsburg 1891, 9-16.

${ }^{4}$ Justinian leitete persönlich die Verwaltung während der Regierung seines Onkels (518-527), weil Justin ein ungebildeter illyrischer Bauer war: Dies wird bekanntlich von Prokop in $A n$. VI.18 f. behauptet, aber auch in De aed. I.3.3, wo unser Historiker Justinian sogar die Bauten zuschreibt, die während der Regierung Justins errichtet

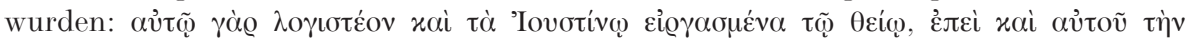

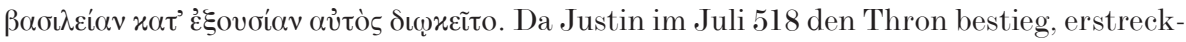
te sich das 32. Jahr der Regierung Justinians stricto sensu vom Juli 449 bis zum Juli 550 .

${ }^{5}$ Cf. die verschiedenen Beiträge über Prokops Bauten in Antiquité Tardive 8 (2000).

${ }^{6}$ G. Greatrex, The Dates of Procopius' Works. BMGS 18 (1994) 101-114 und The Composition of Procopius' Persian Wars and John the Cappadocian. Prudentia 27 (1995) 1-13. Cf. auch jüngst G. Greatrex, Recent Work on Procopius and the composition of Wars VIII. BMGS 27 (2003), im Druck.

7 R. Sсотт, Justinian's Coinage and Easter Reforms, and the Date of the Secret History. BMGS 11 (1987) 215-221 und bes. J.A.S. Evans, The Dates of Procopius' Works: a 
bestimmte Probleme auf, die mit der Datierung zusammenhängen und noch einer Erklärung bedürfen. Es lohnt sich hier also, diesen letzten Aspekt kurz zu behandeln, um jeglichen Zweifel an der Datierung der $A n$ ecdota in das Jahr $550 \mathrm{zu}$ beseitigen.

Der einzige noch heute bestehende Einwand gegen eine frühere Datierung der Anecdota liegt im Prooimion, konkret im ersten Satz des Werkes. Dort erwähnt Prokop seine bisherige Arbeit an den Bella. Laut seiner eigenen Aussage im Prooimion wolle er mit den Anecdota den Bericht der Bella um einiges ergänzen, über das er früher nicht habe sprechen können: ő $\sigma \alpha$

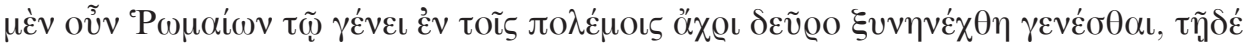

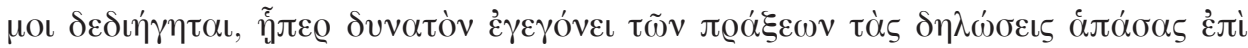

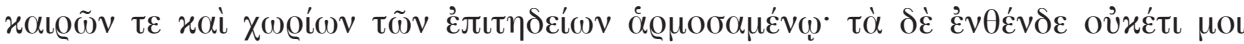

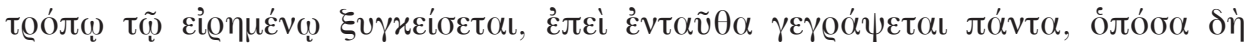

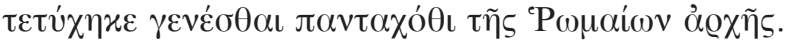

Wenn wir aber in Betracht ziehen, daß die Bücher I-VII der Bella frühestens im Jahre 551 veröffentlicht wurden, da dort die slawischen Plünderungen des Winters 550-51 erwähnt werden ${ }^{8}$, dann wurden die Anecdota vor der Veröffentlichung der Bella geschrieben, falls wir an der früheren Datierung der Anecdota festhalten. Wie kann also Prokop in den Anecdota auf die Bella Bezug nehmen, wenn dieses Werk noch nicht veröffentlicht war? Erstens ist festzuhalten, daß Prokop in diesem ersten Satz der Anecdota keinesfalls ausdrücklich sagt, daß die Bücher I-VII der Bella bereits veröffentlicht waren ${ }^{9}$. Es ist also durchaus möglich, daß Prokop auf die Bücher I-VII der Bella 550 verwies, bevor diese veröffentlicht wurden, aber nachdem sie bereits praktisch abgeschlossen worden waren. Darüber hinaus ist eine wahrscheinliche Hypothese, die bereits Averil Cameron vertre-

Recapitulation of Evidence. GRBS 37 (1996) 301-313. Cf. auch E. KisLinger, Ein Angriff zu viel. Zur Verteidigung der Thermopylen in justinianischer Zeit. BZ 91 (1998) 49-58, bes. A. 56. Der späteren Datierung der Anecdota im Jahre 558 stehen zwei Tatsachen entgegen: 1) Kein Ereignis nach 550 wird in den Anecdota erwähnt (Pace SсотT, Justinian's Coinage, der sogar von Evans, The dates 308-310, widerlegt wird); 2) Die heftige Kritik gegen Theodora in den Anecdota ist nur verständlich, wenn ihr Tod sich kurz zuvor ereignete (Theodora starb bereits 548). Ein möglicher Bezug von An. XVIII.38 auf eine Stelle von De aed., den Evans, a. O. 310-311, postuliert, ist nicht nur sinnlos, sondern größtenteils hypothetisch (für beides ef. J. Signes Codoñer, Procopio de Cesarea. Historia Secreta. Madrid 2000, 48-53).

${ }^{8}$ B. VII.40.33. Evans, The dates 301, bemerkt mit vollem Recht, daß das VII. Buch nach dem slawischen Angriff des Winters 550/551 redigiert worden sein muß, da dieses Ereignis dort erwähnt wird.

9 Das heutige Buch VIII der Bella wurde dagegen nach der Veröffentlichung der sieben ersten Bücher einige Jahre später geschrieben. Cf. unten A. 17. 
ten $^{10}$ und später Geoffrey Greatrex mit guten Argumenten gestützt hat ${ }^{11}$, daß Prokop an den Bella und den Anecdota gleichzeitig arbeitete. Diese gleichzeitige Arbeit an beiden Werken, Anecdota und Bella, wird noch verständlicher, wenn wir bedenken, daß Prokop die heutigen Anecdota ursprünglich als Buch VIII der Bella konzipiert hat. Beweis dafür ist die Tatsache, daß Prokop innerhalb der Anecdota öfters die Bella einfach als „die vorhergehenden Bücher" bezeichnet ${ }^{12}$, während er in seinem späteren Werk Über die Bauten bzw. De aedificiis auf die Bücher der Bella mit Aus-

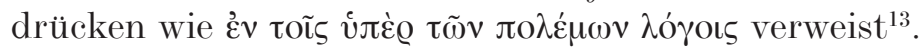

Zusammenfassend kann man also behaupten, daß Prokop 550 Bücher I-VII der Bella um die Anecdota ergänzen wollte, um die acht Bände gemeinsam zu edieren ${ }^{14}$. Er arbeitete möglicherweise seit Jahren an den

${ }^{10}$ Av. Cameron, Procopius and the Sixth Century. London 1985, 9, 52.

11 Greatrex, The composition 6, 10. Greatrex ist der Meinung, daß das durchaus negative Bild von Johannes dem Kappadokier in B. I.25 ursprünglich für die $A n$. bestimmt war, daß aber dieser Abschnitt nach Johannes' Sturz der „offiziellen“ Geschichte beigefügt wurde. Eine weitere Verbindung zwischen dem Inhalt beider Werke hat auch G. Greatrex, Procopius the Outsider?, in: D. C. Sмythe (Hrsg.), From Strangers to Themselves: The Byzantine Outsider. Aldershot (Hampshire) 2000, 215-228, hier 216, hergestellt. Es handelt sich nun um eine sehr ähnliche Bemerkung Prokops in B. I.1.5 und An. I.10 angesichts der $\mu$ o $\theta$ ¡ó (Übeltaten) der Hauptdarsteller seiner Werke: er wolle auch diese Taten seinen Lesern nicht verschweigen. Cf. auch A. 14.

${ }^{12}$ Cf. An. XVI.1; XVI.3; XVII.38; XVIII.14; XVIII.28; XVIII.39; XVIII.44; XX.16; XXI.6; XXIV.23. Nur im ersten Satz des Prooimions verweist Prokop auf die Bella mit

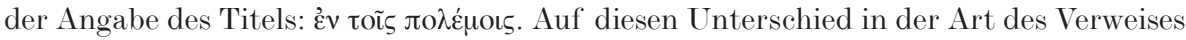
werden wir weiter unten genauer eingehen.

${ }^{13}$ Für derartige Verweise auf die Bella ef. De aed. I.1.6, I.1.12; I.1.21; I.10.3; II.1.4; II.10.10; III.1.12; III.2.8; III.7.7; V.8.2; VI.1.8; VI.5.6 und VI.6.9.

${ }^{14}$ Greatrex, Procopius the Outsider? 219, behauptet, die Anecdota seien eine Art geheimes Dossier mit Materialien, die Prokop nach Justinians Tod in die Bella eingliedern wollte: „Had the emperor succumbed to old age or a plotter's dagger in 549 or 550, it is likely that there would be no Anecdota: all the criticisms that could be incorporated from the Anecdota would have been inserted into the Wars at some point, even at the cost of some coherence to the chronological (and geographical) structure. The resulting work, one might speculate, would have had a far more Herodotean feel than the Wars does, but would undeniably still have been classicizing history". In diesem Fall wären die häufigen Verweise innerhalb der Anecdota auf die vorhergehenden Bücher der Bella als Wegweiser für diese spätere Bearbeitung zu verstehen. Dennoch ist die Struktur der Anecdota keine bloße Exzerptensammlung, sondern weist eine deutliche vierteilige Gliederung auf (cf. Signes, Procopio 37-48 und Anhang am Ende dieses Aufsatzes): 1. Ereignisse an den Kriegsschauplätzen von Persien, Italien und Afrika, die durch das persönliche Verhalten von Belisar, Antonina, Sergios und Salomon zu erklären sind (I-V); 2. Justinian und Theodora während der Regierung von Justin (VI-X.12); 3. Gesamtüberblick über die gemeinsame Regierung von Justinian und Theodora (X.13-XIX); 4. Die Verwaltung des Reiches durch Justinian (XX-XXX). 
Büchern der Bella mit Hilfe der Aufzeichnungen, die er während der Kriege gegen Perser, Vandalen und Ostgoten niedergeschrieben hatte. Dementsprechend dachte Prokop 550 nicht an eine Änderung der Struktur der Bella, die ihm damals sicherlich ziemlich kompliziert erscheinen mußte. Das VIII. Buch sollte also eine Art Appendix zu den Bella bilden und über innenpolitische Ereignisse der Regierung Justinians geheime bzw. gefährliche Informationen liefern, die Prokop während seiner Arbeit in den Bella aus Gründen der Sicherheit verschwiegen hatte ${ }^{15}$. Dies sagt Prokop selbst im

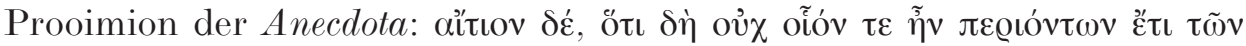

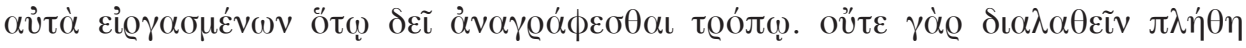

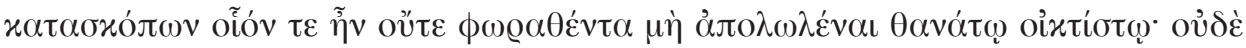

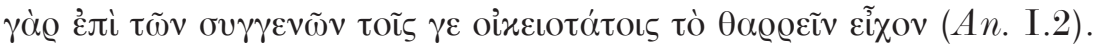

Es wäre jetzt zu fragen, ob die Gefahr, die Prokop früher an solcher Kritik hinderte, aus irgendwelchen Gründen 550 geschwunden war, da Justinian bekanntlich erst 15 Jahre später starb. Doch wollen wir für den Augenblick diese Frage beiseite lassen und zur vorigen Argumentation zurückkehren: Die Verweise der Anecdota auf die 550 noch unedierten Bel$l a$ sind durchaus denkbar, wenn wir annehmen, daß Prokop an beiden Werken gleichzeitig arbeitete und sie sogar als eine Einheit konzipierte. Dennoch ist der erste Satz des Prooimions noch in einer weiteren Hinsicht problematisch. In diesem Satz schreibt Prokop auch, daß er bis jetzt (äx@ı

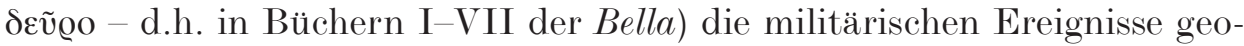
graphisch eingeteilt habe, daß er aber jetzt die späteren Ereignisse ( $\tau \dot{\alpha} \delta \dot{\varepsilon}$ $\grave{\varepsilon} v \theta \varepsilon \dot{\varepsilon} v \delta \varepsilon-$ d.h. nach 550) einfach chronologisch schildern wolle, ungeachtet ihres Schauplatzes. Dies kann nur bedeuten, daß Prokop in den Anecdota über die militärischen Ereignisse nach 550 sprechen wollte. Diese program-

Warum hätte also Prokop, sei es nur als Nachgedanke, die Struktur einer „Geheimgeschichte" herausgearbeitet, wenn er von Anfang an ein einheitliches Werk konzipierte, wo sich innere und äußere Geschichte, Kritik und historische Darstellung mischen sollten? Ist es auch denkbar, daß Prokop diese ,geheimen“ Abschnitte aus dem Kontext der Bella herauslöste, als er dieses letzte Werk 551 veröffentlichte? Ich glaube eher, daß die meiste Kritik der Anecdota keinen Platz in den Bella, sondern nur in einem eigenen Buch finden konnte. Tatsächlich weist der Anfang der Anecdota auf ein abgesondertes Buch hin. Trotzdem muß man die eigentliche Bedeutung von Greatrex' Beitrag anerkennen, der der alten Idee des Gattungsunterschiedes zwischen Anecdota und Bella endgültig ein Ende gesetzt hat. Die Meinung von K. Adshead, The Secret History of Procopius and its genesis, Byz 63 (1993) 4-28, die Anecdota seien eine Sammlung von Materialien, die ein späterer Herausgeber in ein einheitliches Dossier vereint habe, entbehrt jeder Grundlage.

${ }^{15}$ Die Kritik gegen Justinian ist aber bereits in vielen Stellen der Bella zu spüren. Cf. besonders M. Cesa, La politica di Giustiniano verso l'Occidente nel giudizio di Procopio. Athenaeum 59 (1981) 389-409. 
matische Aussage stimmt aber überhaupt nicht mit dem Inhalt der Anecdota überein. Erstens ist die Darstellung der Ereignisse in den Anecdota keineswegs chronologisch; zweitens ist die Struktur der Anecdota für eine geographische Anordnung der Ereignisse völlig ungeeignet, da die meisten Ereignisse in Konstantinopel stattfinden; und drittens erwähnt Prokop kein einziges Ereignis nach $550^{16}$.

Merkwürdigerweise findet sich eine sehr ähnliche Aussage am Anfang des VIII. Buches der Bella, das Prokop Jahre später, ca. 553, veröffentlich$\mathrm{te}^{17}$. Ich gebe hier die Stelle wieder, indem ich die wörtlichen Übereinstimmungen mit dem Anfang der Anecdota fett, und die Anklänge kursiv druk-

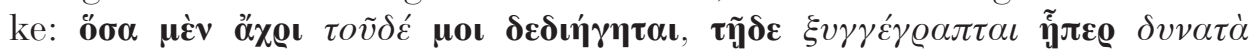

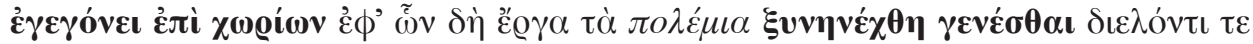

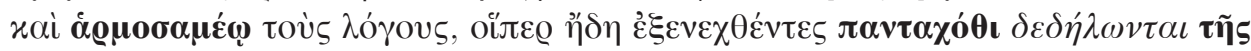
Р⿴囗⿱一一) VIII.1.1).

Die programmatische Aussage am Anfang des VIII. Buches ist hier durchaus angebracht. In den früher veröffentlichten Büchern I-VII hat Prokop die militärischen Ereignisse bis 550 nach den verschiedenen Schauplätzen geschildert, an denen die römischen Heere gekämpft hatten, nämlich Persien, Afrika und Italien. Im VIII. Buch aber schildert Prokop die späteren Ereignisse (bis auf die ostgotische Niederlage am Mons Lactarius im Oktober 552 ${ }^{18}$ ) einfach chronologisch. Prokop hatte schon während der

${ }^{16}$ Der Widerspruch zwischen Prooimion und Inhalt des Werkes wurde bereits von J. Sykoutres, Zu Prokops Anekdota. Textkritisches. BZ 27 (1927) 22-28, notiert.

17 J. A. S. Evans, Procopius. New York 1972, 43 und Evans, The dates 306-308, plädiert aber für eine Datierung des VIII. Buches nach 557. Er stützt sich auf B. VIII.15.16-17, wo Prokop über die Zahlungen der Römer an die Perser spricht. Die Bevölkerung Konstantinopels war laut Prokop gegen diese enormen Zahlungen, die ab 545 liefen und während zweier aufeinanderfolgender Perioden von jeweils 5 Jahren entrichtet werden sollten. Wenn man zu diesen 10 Jahren eine Zwischenperiode von 18 Monate addiert, in denen keine Zahlung vereinbart war, hätten wir also laut Evans das Jahr 556 bzw. 557 als mögliche Abfassungszeit des Werkes erreicht. Eigentlich spricht aber Prokop nur über Vereinbarungen, die noch gültig bleiben. Wenn wir also annehmen, daß der zweite Termin noch nicht überschritten wurde, da Prokop ansonsten über dessen Folgen gesprochen hätte, dann ist das Jahr 556 bzw. 557 eher als terminus ante quem für die Abfassung des Werkes zu betrachten. Neue Argumente für eine Datierung in das Jahr 553 (sogar Ende 552) bringt Greatrex, Recent work, vor, der auf die Tatsache aufmerksam macht, daß die Byzantiner die ganze Zahlung der zweiten fünfjährigen Periode bereits im ersten Jahre entrichteten.

18 Das zuletzt erwähnte Ereignis ist die byzantinische Besetzung von Cumas, die eigentlich erst 553 stattfand. Dieses Ereignis wird aber von Prokop in Verbindung mit dem Feldzug des Jahres 552 gebracht. Greatrex, Recent Work, denkt an eine Folgerung Prokops: „If we suppose that Procopius was writing up this section in Constantinople 
Redaktion der Bücher I-VII der Bella große Probleme gehabt, sich an die geographische Gliederung des Stoffes zu halten. So hatte er die illyrischen Kriege z.B. im Buch der persischen Kriege behandelt, wo er auch über den Nika-Aufstand schrieb. Wenn Prokop den Bericht über die Kriege 553 aktualisiert, verzichtet er endgültig auf diese komplizierte Arbeitsweise und folgt einer konventionellen chronologischen Darstellung. Im Prooimion des VIII. Buches (B. VIII.1.1-2) wird die bereits erfolgte Publikation der Bücher I-VII als Grund für diese neue chronologische Darstellung angeführt, da eine Aktualisierung der jeweiligen Bücher nicht mehr möglich war.

Wenn die programmatische Aussage am Anfang des VIII. Buches angebracht ist, während dieselbe Aussage am Anfang der Anecdota keinen Sinn gibt, folgt daraus, daß die Stelle der Anecdota vom Anfang des VIII. Buches abgeschrieben sein muß, nicht aber umgekehrt, wie es aus der chronologischen Folge der beiden Werke zu schließen wäre ${ }^{19}$. Bereits Sykoutres, der beide Stellen sorgfältig verglich, war zum Schluß gekommen, daß der Anfang der Anecdota nicht in Ordnung ist. Er schlug sogar gewisse Änderungen vor, freilich ohne Nachhall ${ }^{20}$. Er vermerkte besonders, daß der zweite Satz (ab alıı Hinsicht in Einklang zu bringen ist. Was haben nämlich die chronologischen Überlegungen über die Struktur des Werkes in $A n$. I.1 mit der Furcht vor Justinian und Theodora zu tun, die Prokop in An. I.2 als Grund für sein bisheriges Schweigen angibt? Dazu kommt noch der Bezug auf die

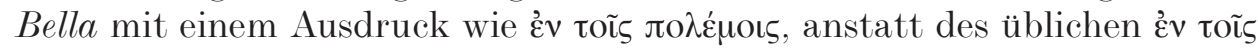

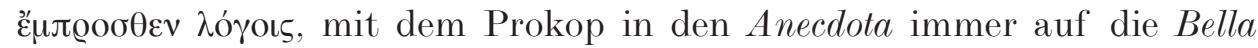
verweist ${ }^{21}$.

Es sieht also aus, als ob der Anfang der Anecdota ein Konzept, nicht ein vollendetes Prooimion wäre. Die Anecdota wurden bekanntlich niemals veröffentlicht, weder während der Regierung Justinians, weil eine solche Edition sehr gefährlich sowohl für Prokop als auch für jeglichen eventuellen Herausgeber gewesen wäre, noch in der Zeit kurz nach dem Tode des Kai-

- the usual view - then such an error is quite understandable, since the terms imposed on the Goths by Narses [i.e. nach Mons Lactarius] demanded that those south of the Po surrender to them". So ist Anfang 553 die wahrscheinlichste Abfassungszeit für Buch VIII (nicht 554, wie es aus Versehen in meinem Buch steht: Signes, Procopios $32)$.

19 So J. B. Bury, History of the Late Roman Empire from the death of Theodosius I to the death of Justinian (395-565). London 1923, 422, A. 2, dem fast alle späteren Gelehrten gefolgt sind.

20 Sykoutres, Zu Prokops Anecdota 22-24.

${ }^{21}$ Cf. A. 12. 
sers, weil in den Werken der klassizistischen Historiker des ausgehenden 6 . und beginnenden 7. Jh.s, die die Bella Prokops intensiv benutzten, keine Spur von der Existenz der Anecdota zu finden ist. Tatsächlich erwähnt keine byzantinische Quelle bis zum 10. Jh. diese „geheime" Geschichte Prokops. Die Suda nennt das Werk zum ersten Mal als Anecdota, „Unedierte Bücher", bei den späteren Autoren wird aber das Werk kaum zitiert. Wir wissen nicht, wie die Anecdota nach den dunklen Jahrhunderten wiederentdeckt wurden, es ist aber wohl möglich, daß der Archetypus der späteren Kopien einen abrupten Anfang aufwies, entweder weil er bereits verstümmelt war ${ }^{22}$, oder weil Prokop das Prooimion der Anecdota nicht fertigstellte, da er dafür auf eine bevorstehende gemeinsame Edition mit den Büchern I-VII der Bella wartete. Irgendjemand hat in byzantinischer Zeit möglicherweise den ersten Satz der Anecdota hinzugefügt, um den fehlenden Anfang zu ergänzen. Dabei ahmte er das Prooimion des VIII. Buches der Bella nach. Es ist aber auch möglich, daß bestimmte Teile des Prooimions des VIII. Buches bereits in die Urschrift der Anecdota eingeflossen waren, da Prokop das Konzept des Werkes nicht für die Publikation bestimmte und es gelegentlich mit Randanmerkungen und etwaigen Notizen versah.

Wie dem auch sei, wenn wir den ersten Satz der Anecdota außer Acht lassen, besteht kein Einwand mehr, die Anecdota in das Jahr 550 zu datieren. Bevor wir aber den Zweck der Anecdota in der politischen Lage dieses Jahres suchen, ist die Möglichkeit zu erwägen, daß sich Prokop durch seinen bevorstehenden Tod zur Abfassung der Anecdota veranlaßt fühlte, wie es Howard Johnston neuerdings vorgeschlagen hat ${ }^{23}$.

\section{Prokops Tod}

Man weiß nicht, wann Prokop starb, da wir über keine äußeren Quellen über sein Leben verfügen ${ }^{24}$. Nur die Datierung seiner Werke kann uns also dabei behilflich sein. Indem wir im vorigen Abschnitt das Buch VIII 553 datiert haben, sind wir somit zu einem vorläufigen terminus post quem angelangt. Wann ist aber Prokops Werk Über die Bauten bzw. De aedificiis zu

${ }^{22}$ Der Anfang des Werkes ist nur im Ambrosianus A 182 aus dem 14. Jh. erhalten. Diese Handschrift ist eine Kopie des Vaticanus gr. 1001 aus demselben Jahrhundert, dessen Anfang verstümmelt und kaum lesbar ist.

${ }^{23}$ J. Howard-Johnston, The Education and Expertise of Procopius. Antiquité Tardive 8 (2000) 19-30.

${ }^{24}$ Die Identität unseres Historikers mit dem gleichnamigen Präfekt des Jahres 562 ist immer noch möglich (cf. Signes, Procopio 16-20), wird aber durch kein Indiz bestätigt. 
datieren? Hier sind die Meinungen der Gelehrten gespalten. Obwohl die neuere Forschung allgemein einer späteren Datierung ca. 559/560 zuneigt ${ }^{25}$, gibt es auch viele Vertreter einer früheren Datierung ca. 554 ${ }^{26}$. Das Problem ist insofern verwickelt, als das Werk, das unvollendet blieb, viele Unebenheiten aufweist, die eine separate Datierung mancher Bücher möglich erscheinen lassen. Im Besonderen hat man häufig eine frühere Datierung des I. Buches über die konstantinopolitanischen Bauten postuliert, da seine vollendete Struktur auf eine selbständige Abfassung hindeuten könnte. Da der 558 stattgefundene Einsturz der Kuppel der Hagia Sophia im I. Buch nicht erwähnt wird, wäre eine frühere Abfassung gegenüber den übrigen, später hinzugefügten Büchern durchaus denkbar ${ }^{27}$. Ich kann hier aus Platzmangel keine der zu diesem Thema vorgebrachten Argumente zur Diskussion stellen, sondern ich werde nur betrachten, welche Folgen die Datierung des De aedificiis für die Deutung der Anecdota mit sich bringt.

Eine spätere Datierung des De aedificiis ca. 560 ist insofern vorteilhaft, als sie uns eine Zeitspanne von zehn Jahren zur Verfügung stellt, die eine persönliche Entwicklung Prokops erklären kann. In zehn Jahren hätte also unser Historiker genügend Zeit gehabt, um von der heftigen Kaiserkritik der Anecdota zur Lobrede Justinians in De aedificiis überzugehen. Falls wir aber De aedificiis in das Jahr 554 zurückdatieren, dann wären dieses Werk und das VIII. Buch der Bella etwa gleichzeitig geschrieben. Da sich Prokop aber im VIII. Buch kritisch über Justinian äußert ${ }^{28}$, oder bestensfalls die Rolle des Kaisers bewußt herunterspielt, schließt die Datierung in das Jahr 554 eine persönliche Entwicklung Prokops als Erklärung für die Verschiedenartigkeit bzw. Widersprüchlichkeit seiner Werke aus. Die Vertreter der

${ }^{25}$ G. Downey, The Composition of Procopius, de aedificiis. Trans. and Proc. of the American Phil. As. 78 (1947) 171-183; Michael Whітвy, Sangarius: Justinian's Bridge over the Sangarius and the Date of Procopius' De aedificiis. Journal of Hellenic Studies 105 (1985) 129-148, und Pride and Prejudice in Procopius' Buildings: imperial images in Constantinople. Antiquité Tardive 8 (2000) 59-66, bes. 63-64; D. Roques, Les constructions de Justinien de Procope de Césarée. Antiquité Tardive 8 (2000) 31-43, bes. 43; E. Jefrreys, Malalas, Procopius and Justinian's Buildings. Antiquité Tardive 8 (2000) 73-79; Signes, Procopio 67-76.

${ }^{26}$ Av. Cameron, Procopius and the Sixth Century. London 1985, 10; Greatrex, The Dates, und neuerdings Howard-Johnston, The Education.

${ }^{27}$ Evans, The Dates. Diese Selbstständigkeit erklärt, daß Buch I häufig getrennt analysiert wird (ef. bes. Mary Whiтву, Procopius' Buildings, Book I: A panegyrical perspective. Antiquité Tardive 8 [2000] 45-57). Demgegenüber hat B. Flusin, Remarques sur la tradition manuscrite du De aedificiis. Antiquité Tardive 8 (2000) 9-17, die früher von Downey vertretene Hypothese von zwei Ausgaben des Werkes überzeugend widerlegt.

${ }^{28}$ B. VIII.26.7 (cf. Signes, Procopio 35-36). 
früheren Datierung versuchten dementsprechend, diese Widersprüche unterschiedlich aufzulösen. So erklärte Averil Cameron 1985 die Unterschiede zwischen den Anecdota und dem De aedificiis als größtenteils durch die Verschiedenheit der Gattungen bedingt, eine Erklärung, die zwar damals nötig erschien, als die Autorschaft Prokops für beide Werke noch umstritten war, die aber heute Cameron selbst in Zweifel zieht ${ }^{29}$. Neuerdings hat Rousseau im De aedificiis die Kritik zwischen den Zeilen wieder lesen wollen und das Werk als einen versteckten $\psi$ ó $\gamma$ os gedeutet ${ }^{30}$, was dem Sinn des Textes Gewalt angetan hat ${ }^{31}$.

Wenn also Prokop tatsächlich gegen seine innere Überzeugung das Werk De aedificiis schrieb, blieb anscheinend nur eine Erklärung übrig: Kaiser Justinian hatte unseren Historiker dazu gezwungen. Downey vertrat als erster kongruent diese Hypothese ${ }^{32}$, die sich bis jetzt größter Beliebheit bei den Gelehrten erfreut und einiges für sich hat ${ }^{33}$. Dennoch hat Howard-Johnston neuerdings eine andere Möglichkeit vorgeschlagen: Prokop habe De aedificiis deswegen geschrieben, weil er sich von seinem Beruf her für die Bautechnik interessierte. Laut diesem Gelehrten wäre Prokop kein Jurist, wie es normalerweise angenommen wird, sondern eher ein Ingenieur $^{34}$. Obwohl Howard-Johnston sowohl die offenkundige Kompetenz Prokops im Heerwesen als auch seine juristische Ausbildung außer Acht gelassen $^{35}$, den offiziellen Charakter der meisten von Prokop benutzten

29 Av. Cameron, Conclusion. Antiquité Tardive 8 (2000) 177-180, bes. 178: „I am less certain now than I was at the time of the publication of my book on Procopius in 1985 that the answer to these problems is to be found in literary genre".

${ }^{30}$ P. Rousseau, Procopius's Buildings and Justinian's Pride. Byz 68 (1998) 121-130.

${ }^{31}$ Cf. Michael Whitby, Pride and Prejudice.

32 Downey, The Composition 181-183.

${ }_{33}$ Daß es sich um einen Auftrag handelt, bezeugt Prokop vielleicht in De aed. I.3.1, wo

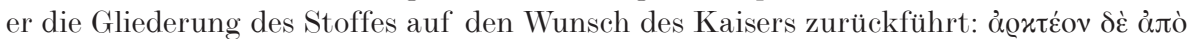

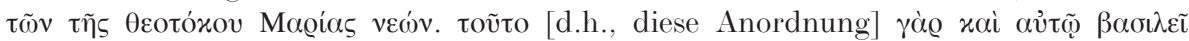

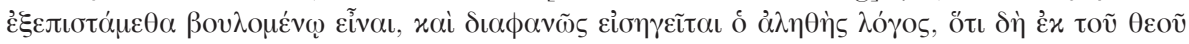

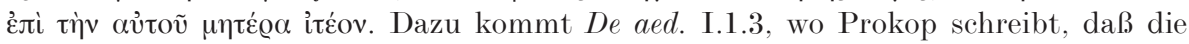
Geschichte die Dankbarkeit der Untertanen gegenüber ihren Herrschern bezeugt. Howard-Johnston, The Education and Expertise 25, A. 29, ist der Meinung, daß beide Stellen keineswegs einen kaiserlichen Auftrag beweisen. Er hat vielleicht Recht, ich finde dennoch ohne einen solchen Auftrag keinen Grund für eine frühere Abfassung des Werkes im Jahre 554. Die amtlichen Quellen, die Prokop für seine Arbeit benutzt, weisen dazu eindeutig auf einen offiziellen Auftrag hin. Die wiederholte Erwähnung der kaiserlichen Gnade gegenüber den Verschwörern in De aed. I.1.10 und 16 könnte auch auf persönliche Gründe hinweisen (cf. auch A. 54).

${ }^{34}$ Howard-Johnston, The Education and Expertise.

${ }^{35}$ Für die erstere ef. dagegen W. KaEgi, Procopius the military historian. BF 15 (1990) 53-85 und Greatrex, Recent work; für die zweite ef. Signes, Procopio 10-12, 95-100. 
Quellen nicht genügend berücksichtigt ${ }^{36}$, und schließlich die Kritik gegen die justinianische Baupolitik in den Anecdota heruntergespielt hat ${ }^{37}$, ist seine frühere Datierung des De aedificiis im Jahre 554 mit einer neuen Deutung der Anecdota verbunden, die uns hier unmittelbar betrifft.

Howard-Johnston hat den unvollständigen Zustand sowohl des VIII. Buches der Bella und des De aedificiis als auch der Anecdota mit dem vorzeitigen Tod Prokops verbunden, den er 553 datiert:

There is only one plausible explanation for Procopius' sudden abandonment of the richly textured military history over which he had laboured for so many years and which he was taking care to keep up to date [...] He was incapable of carrying on. Death, of which he reveals some intimations in the preface and hastly written final sentence of the Secret History, is the most likely cause of this incapacity. We thus acquire our final piece of information: it is highly likely that Procopius died in the course of 553. It follows that he stopped work by that time both on the evidently unfinished Secret History and on the Buildings, which may have a formal conclusion (6, 7, 17-20), but is very far from being complete. [...] He died in 553, perhaps after an illness which gave him time to top and tail the Secret History, to write a perfunctory conclusion to the Buildings, and to bring the Wars to a rather scrambled close at the end of $552^{38}$.

Ich bin nicht davon überzeugt, daß nur ein vorzeitiger Tod den unvollendeten Zustand von Prokops Werken erklären kann. Was die Anecdota betrifft, werden wir gleich unten andere (ergänzende) Möglichkeiten in Betracht ziehen. Bella I-VII wurden 551 mit einem unpassenden Ende publiziert, genau so wie Bella VIII zwei Jahre später. Die Abwesenheit Prokops vom Kriegsgebiet ist auch eine befriedigende Erklärung für den Mangel an einer Fortsetzung der Bella. Ein offizieller Auftrag hätte sicherlich die Arbeit Prokops am De aedificiis ziemlich erschwert; vielleicht verzichtete er deswegen auf seine Vollendung und setzte frühzeitig dem Werk ein Ende. Da die Datierung des De aedificiis umstritten ist, baut HowardJohnston seine Hypothese auf einer sehr wackeligen Basis. Dennoch hat er mit seiner Hypothese eigentlich nur auf die in der Forschung immer

${ }^{36}$ Wenn die persönliche Erfahrung Prokops die Auswahl und Schwerpunkte des De aed. entscheidend mitbestimmt hat, warum sind dann die italienischen Bauten nicht in diesem Werk vertreten, obwohl Prokop die justinianische Eroberung Italiens miterlebte? Könnte dieses Fehlen nicht aus Mangel an offiziellen Berichten über die italienischen Bauten erklärt werden, da Italien über eine autonome Verwaltung innerhalb des justinianischen Reiches verfügte? Die Beiträge über De aedificiis in Antiquité Tardive 8 (2000) lassen deutlich die Verbindungen zwischen der justinianischen Propaganda und De aedificiis in den Vordergrund treten. Ich werde demnächst diesem Problem einen gesonderten Beitrag widmen.

${ }^{37}$ Cf. $A n$. VIII.7-8; XI.3; XVIII; XIX.6, 15; XXIII.6-7; XXIV.12-13; XXVI.7, 23-25, 33, und Roques, Les constructions 34, A. 21.

38 Howard-Johnston, The Education and Expertise 21-22. 
wiederkehrende Frage nach dem möglichen Empfänger der Anecdota beantworten wollen: An welche Leser waren denn die Anecdota gerichtet?

Howard-Johnston glaubt, daß Prokop in An. I.5 und XXX.34, ,assumes that contemporaries of his, including Justinian himself, will outlive him"39, so daß er anscheinend das Werk für einen Leserkreis bestimmte, der sich nur nach seinem Tod sammeln könnte. Die von Howard-Johnston angeführten Beweisstellen sind hier nun näher zu betrachten.

In $A n$. I.5 spricht Prokop seine feste Überzeugung aus, daß seine Zeitgenossen den künftigen Generationen die Wahrheit übermitteln würden. Dies ist aber nicht unbedingt im Sinne einer posthum gedachten Ausgabe zu deuten, für die ich eigentlich in der Antike keine Parallele gefunden habe, sondern kann einfach als ein historiographischer Topos gewertet werden, in dem Prokop seine Hoffnungen äußert, von seinen Zeitgenossen auch künftig bestätigt zu werden. Prokop schreibt wie üblich in der griechischen Geschichtsschreibung für künftige Leser und Generationen, dies bedeutet aber nicht, daß er auf eine Ausgabe seines Werkes bereits während seines Lebens verzichtet hat.

Es ist zwar durchaus richtig, daß er am Ende der Anecdota (XXX.34) behauptet, daß die Wahrheit seiner Behauptungen erst nach Justinians Tod bestätigt werden würde. Prokop datiert dazu das Werk viermal ausdrücklich in das 32. Jahr der Regierung Justinians, wobei die Gegenwart betont wird. Dennoch schreibt Prokop vom Kaiser immer in der Vergangenheit, als ob er bereits tot wäre. Der Tod des Kaisers ist sogar eine Voraussetzung für die Publikation des Werkes, da Prokop im Prooimion schreibt, daß erst der Tod der Hauptdarsteller seiner Geschichte (in erster Linie Justinian!) die Abfassung des Werkes ermöglicht hat (An. I.2). Einige Zeilen später ( $A n$ I.8) spricht Prokop sogar vom bereits eingetretenen, anscheinend abschreckenden Tod des kaiserlichen Ehepaares (ő $\pi \varepsilon \varrho x \alpha i$

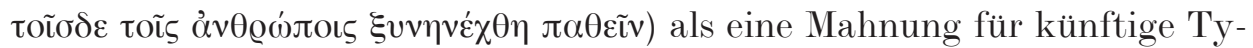

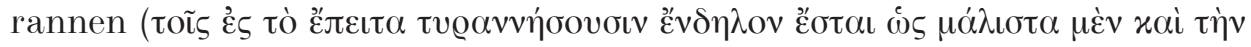

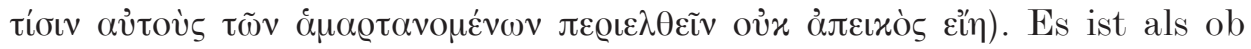
Prokop anfänglich vom nahe bevorstehenden Tod Justinians überzeugt wäre und sich mit dieser Hoffnung ans Werk gemacht hätte. Nur im letzten Satz des Werkes hätte unser Historiker festgestellt, daß Justinian noch lebte und dann hinzugefügt, daß erst Justinians Tod die Wahrheit seiner Darstellung bestätigen könnte. Es gibt eine Spannung zwischen der Zukunft ohne Justinian, für die Prokop schreibt, und der Gegenwart mit dem Kaiser, der immer noch am Leben ist. Diese Spannung erklärt die angemerkten Widersprüche, bedeutet aber nicht, daß Prokop etwa seinen nahen

${ }^{39}$ Howard-Johnston, The Education and Expertise 21, A. 12. 
Tod geahnt hätte. Wäre dies der Fall, warum hätte unser Historiker dann eine Lobrede auf Justinian wie De aedificiis ein paar Jahre später geschrieben? Wenn er tatsächlich nur über Bautechnik sprechen wollte (so HowardJohnston), so hätte er ein Enkomion dafür nicht nötig gehabt! Ich denke demzufolge, daß die Gründe für die Abfassung der Anecdota in der politischen Lage des Jahres $550 \mathrm{zu}$ suchen $\operatorname{sind}^{40}$ und nicht im mutmaßlichen, unmittelbar bevorstehenden Tod seines Verfassers.

\section{Germanus und die Nachfolge Justinians}

Die Geschehnisse des Jahres 550 werden am Ende des VII. Buches der Bella behandelt. Der General Germanus, Justinians Vetter, steht dabei im Mittelpunkt der Erzählung Prokops. Er wird damals von Justinian zum Oberbefehlshaber gegen die Ostgoten unter Totila ernannt und sammelt, größtenteils aus eigenen Mitteln, ein beträchtliches Heer, mit dem er, so Prokop, ganz Italien für das Reich wiedergewinnen wollte ${ }^{41}$. Mit seiner jüngst geschlossenen Ehe mit Matasuntha, der Tochter Amalasuntas und Enkelin Theoderichs, erhoffte sich Germanus, viele gotische Truppen auf die römische Seite ziehen zu können ${ }^{42}$. Tatsächlich schreibt Prokop, daß sich viele ostgotische Kontingente davor fürchteten, das „Geschlecht von

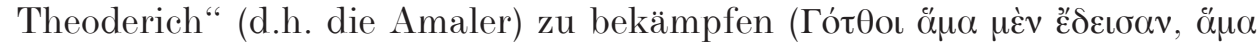

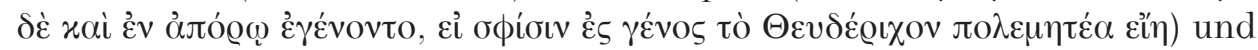
sogar bereit waren, die Seiten zu wechseln ${ }^{43}$. Die römischen Truppen in Italien, die bis dahin völlig demoralisiert zerstreut im Land hin und her

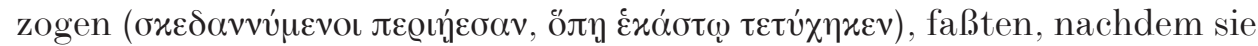
die Nachricht von Germanus' Ankunft hörten, wieder Mut und leisteten erneut Widerstand gegen die ostgotischen Angriffe ${ }^{44}$. Zu diesem Zeitpunkt (\$29) endet der Winter und Prokop schließt thukydideisch seine Erzählung: „Dies trug sich so zu. Der Winter ging dann zu Ende und somit hörte das

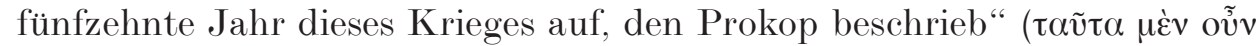

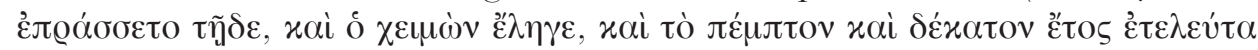

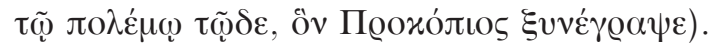

${ }^{40}$ Ich lasse hier die religiösen Befürchtungen und Endzeiterwartungen Prokops beiseite, die mit der teuflischen Identität Justinians verbunden waren und sich nach Theodoras Tod zuspitzten. Diese Probleme, die sicherlich eine Rolle für die Absfassung der Anecdota spielten, werde ich in einem gesonderten Beitrag behandeln.

${ }^{41}$ B. VII.37.24 y $39.9-13$ y $16-20$.

${ }^{42}$ B. VII. 39.14-15. Die Hochzeit fand wahrscheinlich 549 statt.

${ }^{43}$ B. VII.39.21-22.

${ }^{44}$ B. VII.39.23-28. 
Diese Stelle bezeichnete ursprünglich vielleicht das Ende des VII. Buches der Bella. Prokop schrieb diese Zeilen nach Ende des Winters und des 15. Jahres seiner Geschichte, also im Frühling 550. ${ }^{45}$ Möglicherweise erhoffte er sich einen triumphalen Einzug des Germanus in Italien für den Sommer dieses Jahres. Da unser Historiker zu diesem Zeitpunkt, wie oben gezeigt, gleichzeitig an den Anecdota arbeitete, kann man vermuten, daß ein Anschluß an die Anecdota ursprünglich nach dem Ende dieses Kapitels 39 vorgesehen war. Die Hoffnungen von Prokop waren also 550 mit großer Wahrscheinlichkeit nicht nur auf einen Sieg des Germanus in Italien, sondern auch auf einen nach diesem Sieg zu erwartenden Kurswechsel in der Regierung gerichtet. Wie sich dieser Kurswechsel verwirklichen hätte können, werden wir später sehen. Für den Augenblick reicht es uns hier zu sagen, daß Prokop ein passendes provisorisches Ende für seine Darstellung in den militärischen Vorbereitungen für den Einmarsch in Italien sah.

Etwas war jedoch inzwischen geschehen, das Prokops Hoffnungen völlig niederschmetterte: ein Einfall der Slawen, die unerwartet die Donau überquerten und nach Naïssus zogen. Die Slawen hatten sogar vor, Thessalonike einzunehmen. Da Germanus die Aushebung der Truppen für Italien im benachbarten Serdica ausführte, befahl ihm Justinian, gegen die Feinde zu ziehen. Es kam jedoch nie zu einem Zusammenstoß zwischen den Slawen und Germanus: Als die Slawen erfuhren, daß Germanus gegen sie ziehen wollte, gingen sie nach Dalmatien (also nach Westen) und räumten das Feld. Germanus konnte wieder an seinen Einmarsch in Italien denken, aber er starb unerwartet zwei Tage vor dem vorgesehenen Termin an einer unbestimmten Krankheit. Damit waren auch Prokops Hoffnungen erloschen.

Prokop fügte trotzdem seiner früheren Darstellung, die wie gesagt provisorisch mit Kapitel 39 aufhörte, die Schilderung dieser Ereignisse hinzu. Diese Ergänzung bildet das heutige Kapitel 40, das letzte Kapitel des VII. Buches, wo auch über die Folgen von Germanus' Tod gesprochen wird. Erstens mußte die römische Flotte, die das von den Goten belagerte Syrakus erreicht hatte, diese Stadt verlassen, da sie sich ohne den nötigen Nachschub dort nicht mehr behaupten konnte. Zweitens waren die römischen Truppen in Dalmatien, die von Johannes und Justinian (zwei Ver-

${ }^{45}$ Prokop läßt das Jahr unter Berufung auf die Formel des Thukydides immer nach dem Winter beginnen. Da der nach dem Winter eintretende Sommer für Thukydides auch den Frühling einschließt (A. W. Gonne, A historical commentary on Thucydides, Bd. III, Oxford 1956, S. 699-715: "Appendix on summers and winters"), bildet März für beide Historiker den Jahresanfang. Für die damals üblichen alexandrinischen und protobyzantinischen Ären begann das Jahr auch Ende März (am 21. bzw. 25.), wieder mit dem Ende des Winters (V. Grumel, La chronologie, Paris 1958, S. 73-97 und 111-128). 
wandten des Germanus) geführt wurden, von den Slawen besiegt worden, obwohl die meisten römischen Gefangenen gleich danach befreit werden konnten. Damit endet das VII. Buch.

Daß die Schilderung von Germanus' Tod und von den unmittelbar danach stattgefundenen Geschehnissen ein Nachtrag Prokops war bzw. daß Prokop Buch VII mit Kapitel 39 schließen wollte und es nur nachträglich um Kapitel 40 ergänzte, ist meiner Meinung nach durch den Umstand bewiesen, daß er in diesem Kapitel 40 die Schilderung der Ereignisse des 16. Jahres seiner Kriegsgeschichte nicht fertigstellte, sondern vorzeitig enden ließ, um sie erst in dem zwei Jahre später geschriebenen Buch VIII

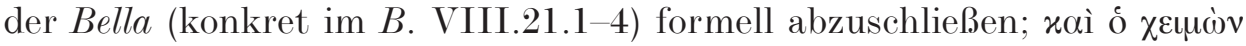

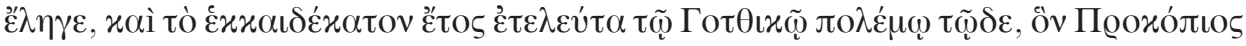
$\xi \cup v \varepsilon ́ \gamma \varrho \alpha \psi \varepsilon$. Die Ereignisse zwischen Frühling und Winter 550 werden dazu in B. VII.40 sehr kurz geschildert und beschränken sich hauptsächlich auf den unerwarteten Tod des Germanus und dessen Folgen. Da die Anecdota während des 32. Jahres der tatsächlichen Regierung Justinians (d. h. zwischen Juli 549 und Juli 550) redigiert wurden, ist es möglich, daß Germanus' Tod erst im Sommer bzw. Herbst 550 stattfand, als Prokop die sieben ersten Bücher der Bella (mit Ausnahme von VII.40) bereits abgeschlossen und den größten Teil der ursprünglich als Buch VIII gedachten Anecdota geschrieben hatte.

Der unerwartete Tod des Germanus hat auch eine interessante Laudatio des Generals in Kapitel 40.9 verursacht, wo Prokop seine Bewunderung nicht verhüllen kann. Dieses Porträt des Germanus ist genau das Gegenstück zum Bild Justinians in den Anecdota, wie bereits einige Gelehrte en passant bemerkt haben ${ }^{46}$. Es handelt sich um ein richtiges é $\gamma$ rópuov des

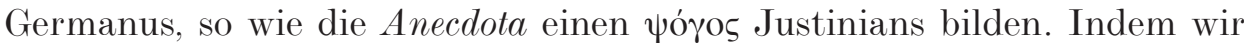
den Text in sieben Abschnitte gliedern und diese den entsprechenden Stellen des Porträts Justinians in den Anecdota gegenüberstellen, wird der Gegensatz zwischen den beiden Figuren offensichtlich. Nochmals rufen wir in Erinnerung, daß beide Werke, wie oben gezeigt, zur gleichen Zeit geschrieben wurden:

1. Selbständigkeit versus Beeinflußbarkeit: Germanus $(=G)$ war ein selbständiger General

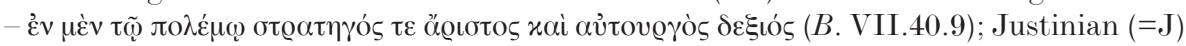

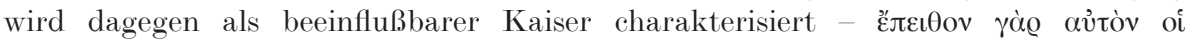

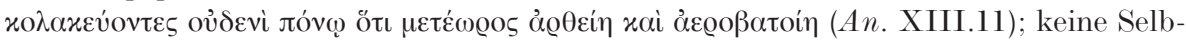

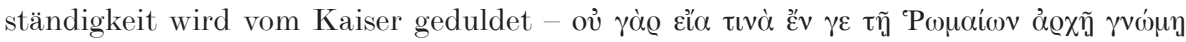

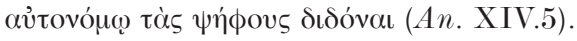

${ }^{46}$ So B. Rubin, Prokopios von Kaisareia, RE XXXIII, Sp. 502-503. Cf. auch Av. Cameron, Procopius 54, 140-141 und Evans, Procopius 104-105. 
2. Beobachtung der Gesetze: G. wird wegen seiner strengen Beobachtung der Gesetze

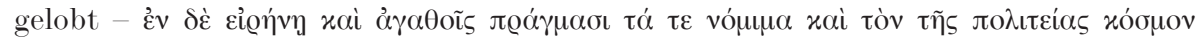

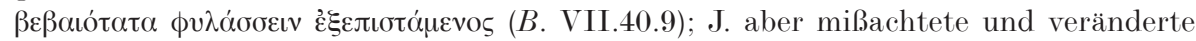

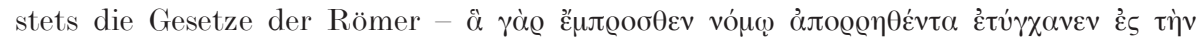

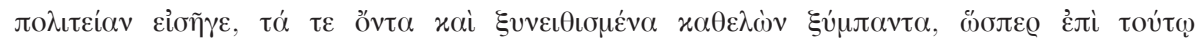

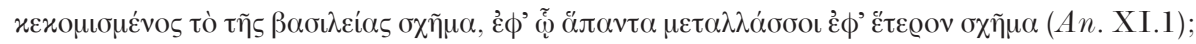

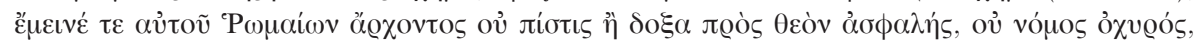

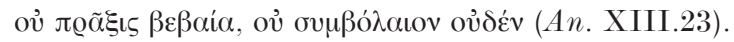

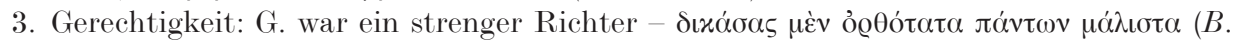

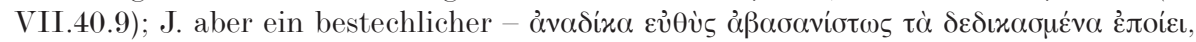

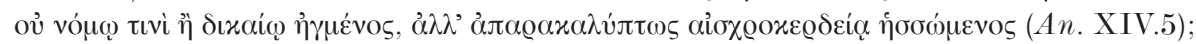

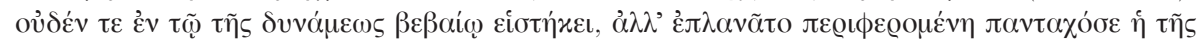

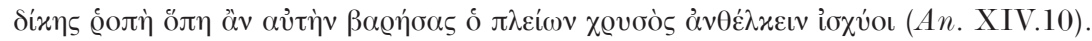

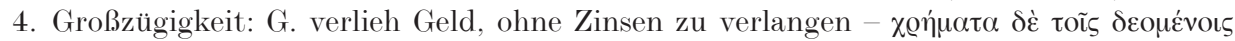

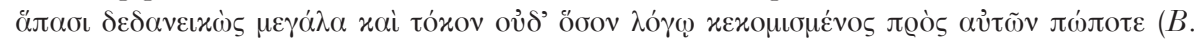

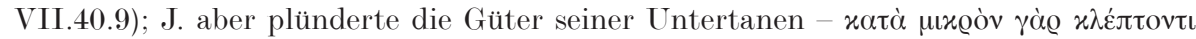

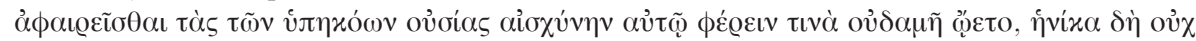

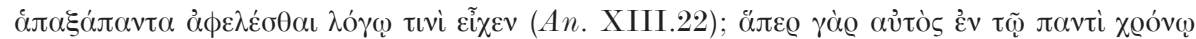

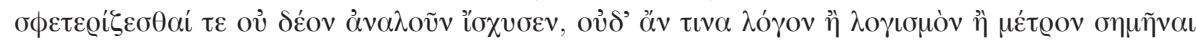

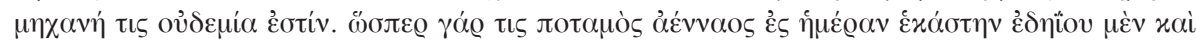
$\dot{\varepsilon} \lambda \eta \hat{i} \zeta \varepsilon$ to toù

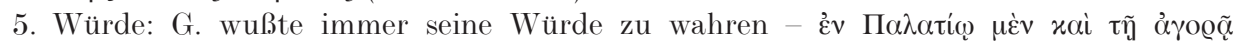

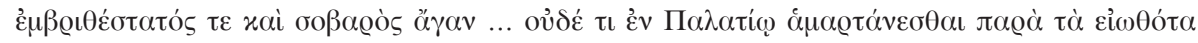

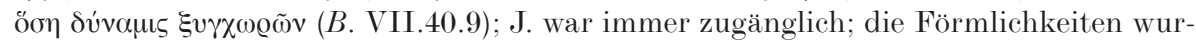

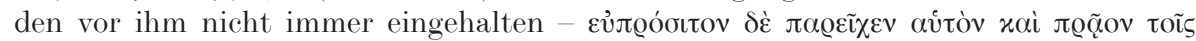

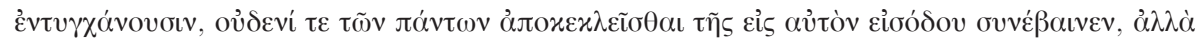

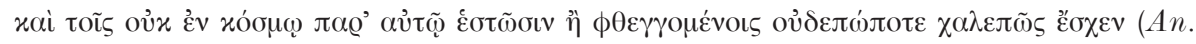
XIII.1-2).

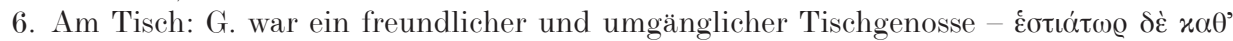

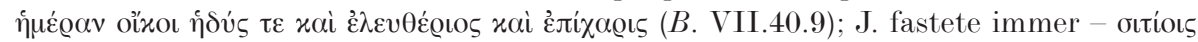

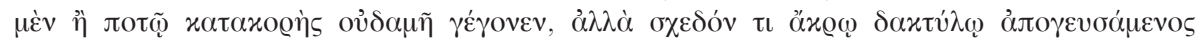

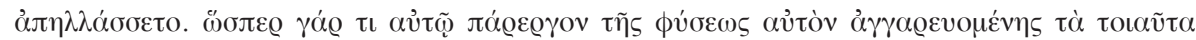

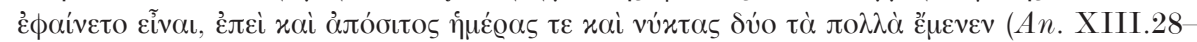

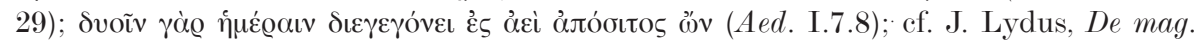

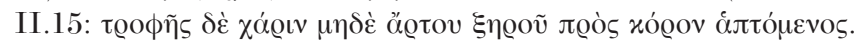

7. Verbindung mit den Zirkusparteien: G. lehnt jeden Kontakt mit den aufwieglerischen

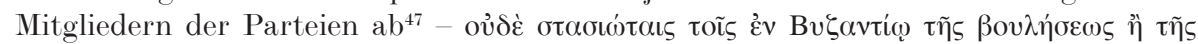

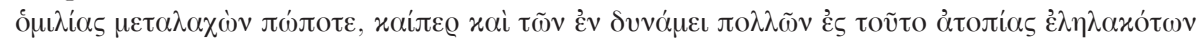

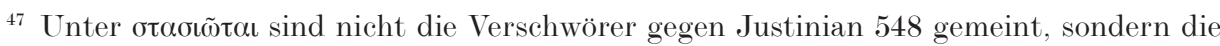
Zirkusparteien, wie es der Vergleich mit $A n$. VII deutlich macht, wo die Parteimitglie-

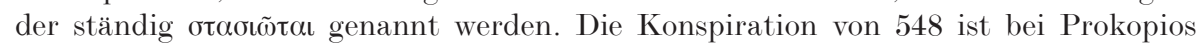

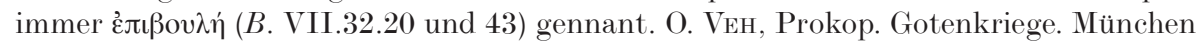
1961, 705 hat die Stelle richtig übersetzt: ,,[Germanus] duldete, soweit dies in seiner Macht lag, am Kaiserhof keine Verstöße gegen die herkömmliche Ordnung und nahm niemals Anteil an den Bestrebungen der Parteien in Byzanz, ja mied sogar den Verkehr mit ihnen, wiewohl sich schon viele einflußreiche Männer auf solche Torheit eingelassen hatten." 


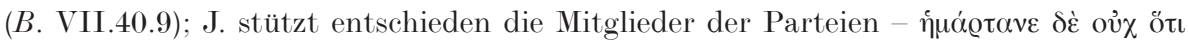

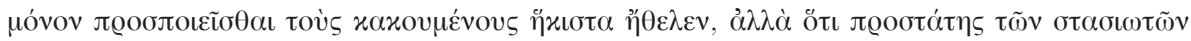

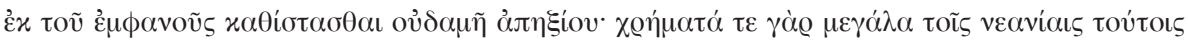

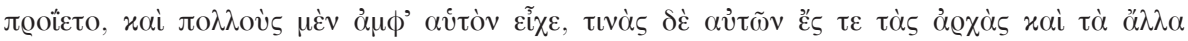

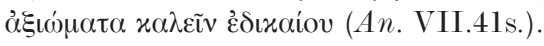

Es ist eine Schwarz-Weiß-Malerei, wobei selbstverständlich Justinian das Schwarze und Germanus das Weiße zukommt. Interessant ist besonders der siebte Abschnitt. Laut Prokop hielt sich Germanus von den Zirkusparteien, die am Hof eine gewisse Stütze fanden, fern. Mit dieser Stütze ist hier sicherlich Justinian gemeint, da Prokop im Kap. 7 der Anecdota ausführlich beschreibt, wie die Zirkusparteien einen starken Rückhalt in Justinian fanden, der Ihnen sogar Hofwürden verlieh.

Mit dieser Laudatio des Germanus verdeutlichte Prokop die Mängel des gehaßten Justinian. Ein ähnliches Verfahren wird in den Bella gelegentlich benutzt, wenn Prokop seine eigene Kritik an Justinian, die in den Anecdo$t a$ explizit wird, in den Mund der Feinde des Reiches legt ${ }^{48}$. Mit der Figur des Germanus aber waren auch, wie oben angedeutet, die Hoffnungen Prokops auf einen Kurswechsel in der Regierung verbunden. Es handelt sich also nicht nur darum, daß Prokop diese Laudatio des Germanus als Mittel für die Kaiserkritik symbolisch ausgestaltet hat, sondern auch daß er möglicherweise persönlich gewisse Interessen bzw. Kontakte mit dem Kreis um Germanus hatte. Ein Beweis dafür ist seine Beschreibung der Verschwörung des Artabanes gegen Justinian, in die Germanus verwickelt war. Prokopios schildert ausführlich diese 548 stattgefundene Verschwörung am Ende des VII. Buches. Wir wissen nicht, ob dieser Abschnitt vor oder nach Germanus' Tod geschrieben wurde, es wird aber durch eine kursorische Lektüre sofort offenkundig, daß unser Historiker in vieler Hinsicht auf der Seite der Verschwörer gestanden haben dürfte. Einige Punkte sind hier hervorzuheben.

Artabanes, der Hauptverschwörer, ist Prokop nicht unsympathisch. Unser Historiker erzählt, daß Kaiserin Theodora die Ehe des Artabanes mit Preiecta (einer Nichte Justinians) verhinderte, da Artabanes noch eine Frau hatte, die er aus unbekannten, aber durchaus üblichen Gründen vie-

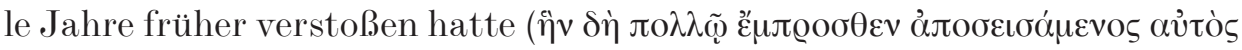

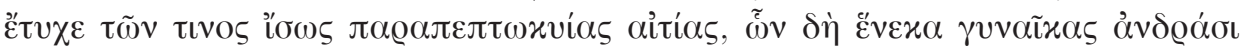

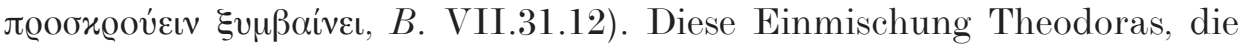
sogar die Ehe Preiectas mit Joannes, dem Enkel des Hypatius, arrangierte

${ }^{48}$ Cf. die Rede der ostgotischen Botschafter vor Chosroes in B. II.2.12 und die Rede Totilas gegen Justinian in B. VII.21.12-17. Cf. also Signes, Procopio 35. 
(B. VII.31.14 $)^{49}$, hat Artabanes sehr verärgert, da er seine Liebe von Preiecta erwidert glaubte. Er rechnete sich auch seinen siegreichen Feldzug in Afrika als großen Verdienst an und betrachtete seine erzwungene Ehe mit seiner verstoßenen Frau als unangebracht (B. VII.31.15). Prokop schreibt dazu: „eine Situation [d.h., die unerwünschte Ehe], die die Seele eines

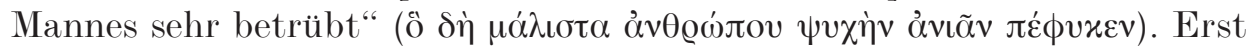

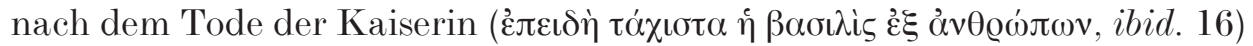
konnte sich Artabanes von seiner Frau trennen.

Die Kritik gegen die Heiratspolitik Theodoras begegnet uns auch in den Anecdota, wo ähnliche Fälle beispielhaft behandelt werden. Sympto-

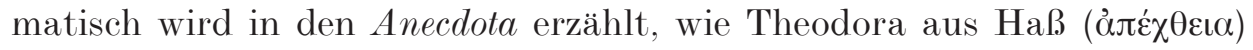
gegen Germanus die Verlobung von dessen Kindern verhinderte. Germa-

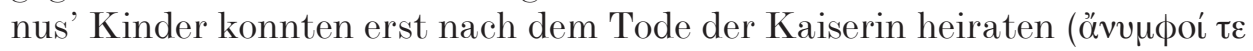

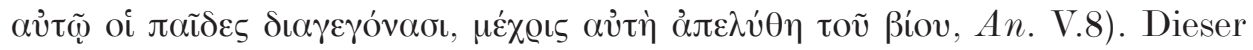
Punkt wird von Prokop in den Bella sicherlich aus Vorsicht nicht behandelt. Dennoch erwähnt Prokop Germanus' Ärger über Theodora, als diese gegen den letzten Willen seines verstorbenen Bruders Boraides die Rechte von dessen Tochter gegenüber denen der übrigen Familie verteidigte (ő $\varepsilon \varrho$

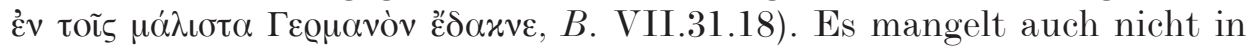
den Anecdota an Kritiken gegen Theodora und Justinian wegen ihrer parteiischen Handhabung der Erbschaften. Es ist also nicht zufällig, daß Prokop die Ursachen für die Verschwörung des Artabanes mit diesen anscheinend privaten Angelegenheiten verbindet. Eigentlich handelte es sich nicht um private Angelegenheiten, sondern, wie wir unten sehen werden, um Machtverteilung innerhalb der mächtigsten Familien des Reiches.

Die nächste Figur in der Verschwörung ist Arsakes, ein Verwandter von Artabanes, der ihn zum Aufruhr anstiftet. Prokop erteilt Arsakes das Wort, der vor uns ungehindert als ausgezeichneter Feind Justinians spricht, also eigentlich wie Prokop selber gesprochen hätte ${ }^{50}$. Arsakes schildert zuerst seinem Freund Artabanes die schlimme Lage des römisch besetzten Armenien (ihr gemeinsames Vaterland) und die Leiden ihrer Familen ( $B$. VII.32.7). Die Anspielung auf die schweren Abgaben, die Armenien an die Römer liefern muß, erinnert an ähnliche Beschwerden in den Anecdota gegen die finanzielle Unterdrückung im justinianischen Reich. Die persönlichen ehelichen Probleme des Artabanes seien, setzt Arsakes fort, nicht damit zu vergleichen. Es wäre also nötig, Justinian zu ermorden, eine an

${ }^{49}$ Wir können nur über die Gründe spekulieren, welche Theodora zu dieser Eheschließung Preiectas mit Johannes bewogen. Zu Johannes cf. Prosopography of the Later Roman Empire (=PLRE) IIIA, s.v. Ioannes 63.

${ }^{50}$ Cf. A. 48. 
sich unproblematische Aktion, da der Kaiser mitten in der Nacht (ảw@i

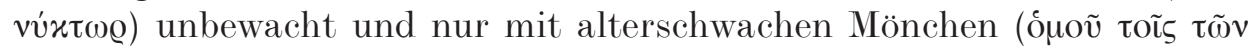

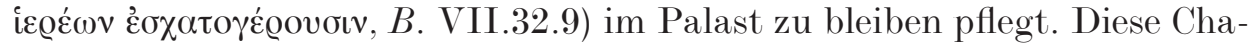
rakterisierung Justinians ist wieder in den Anecdota zu finden, wo diesmal Prokop selbst sagt, daß der Kaiser nur ein böser Dämon sein könne, da er

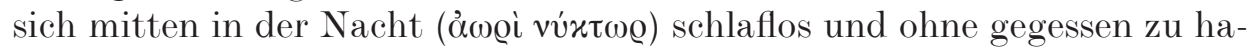
ben in den Gemächern des Palastes herumirre (An. XII.27). Daß Mönche die nächtlichen Begleiter Justinians waren, ist auch aus An. XII.20 zu entnehmen, wo Prokop aber die Mönche durch eine Umschreibung als Per-

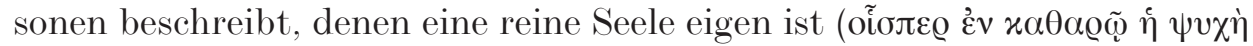
ๆ๊v). Wenn Prokop in B. VII.35.11 schreibt, daß sich Justinian den theologischen Streitigkeiten widmete und kaum um die politische Lage Italiens kümmerte, hat er möglicherweise dieselbe Szene im Kopf. Zusammenfassend kann man also behaupten, daß Arsakes und Prokop eine ähnliche Meinung über Justinian hatten.

Nach Artabanes und Arsakes wird der dritte Verschwörer, Chanaranges, ein armenischer Freund des Arsakes, kurz vorgestellt (B. VII.32.12). Die drei Armenier versuchen nun, den Germanus durch seinen Sohn Justinus für ihre Pläne zu gewinnen (B. VII.32.13-21). Justinus lehnt ihre Vorschläge ab und teilt seinem Vater alles mit. Germanus spricht dann mit Markellos, dem Befehlshaber der Palastwachen (B. VII.32.22-23), der seinerseits Vorsicht für angebracht hält und Beweise fordert, bevor man die Angelegenheit vor den Kaiser bringt (B. VII.32.23-26). Ein Treffen des Justinus mit Chanaranges folgt. Dieser führt ein Gespräch mit Artabanes und bestätigt dann gegenüber Justinus die Ziele der Verschwörer (B. VII.32.27-31). Danach begegnet Germanus selbst dem Chanaranges, der wiederum die Pläne der Verschwörer darlegt: Germanus solle der künftige Kaiser sein, nachdem Justinian, Belisar und Markellos beseitigt seien; da Belisar, der bereits Italien verlassen habe, noch nicht in Konstantinopel angekommen sei, müßten die Verschwörer auf seine Anwesenheit in der Hauptstadt warten, bevor sie ihre Pläne in die Tat umsetzten. Leontios, ein Freund des Germanus, hört versteckt zu (B. VII.32.32-40) und teilt alles dem Markellos mit (B. VII.32.40). Auch Germanus informiert seine Freunde Bouzes und Konstantianos über sein Gespräch mit Chanaranges (B. VII.32.41).

Bis jetzt haben wir also neun Personen, die in der einen oder anderen Weise von der Verschwörung unterrichtet sind. Markellos und Germanus sammelten zu diesem Zeitpunkt genügend Beweise, um die Sache vor den Kaiser zu bringen. Trotzdem bleiben sie weiterhin untätig. Markellos ist laut Prokop immer noch unsicher und fürchtet, daß Artabanes aufgrund

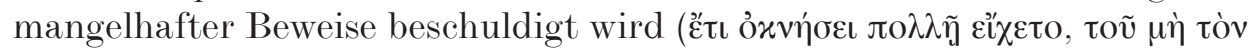




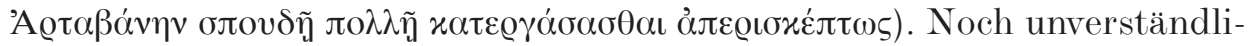
cher ist das Verhalten von Germanus, der seinen beiden Freunden die

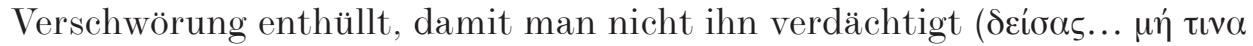

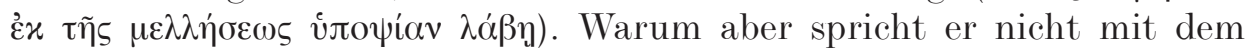
Kaiser selbst, um diese Gefahr zu vermeiden? Worauf warten sie beide?

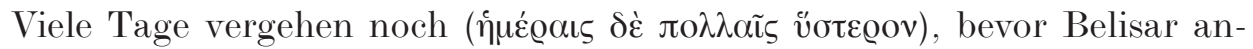
kommt. Erst dann enthüllt Markellos dem Kaiser die ganze Verschwörung (B. VII.32.42).

Etwas stimmt anscheinend nicht. Man hat trotz des Berichtes Prokops den Eindruck, daß Germanus und vielleicht auch Markellos nicht genügend Abstand von der Verschwörung genommen hatten, daß sie sogar mit den armenischen Anstiftern gemeinsame Sache machten ${ }^{51}$. Warum haben sie aber die Verschwörung nicht in die Tat umgesetzt? Sie verfügten wahrscheinlich über Sympathien in den Machtkreisen der Hauptstadt. Tatsächlich hat der Senat Germanus trotz der gefährlichen Beschuldigungen von jeglicher Schuld freigesprochen. Artabanes selbst, obwohl für schuldig erklärt, wurde nur im Palast unter Hausarrest gestellt (B. VII.32.51) und kurz danach vom Kaiser mit eigenen Truppen nach Sizilien geschickt ( $B$. VII.39.8-9). Justinian war anscheinend, so Prokop, mit dieser Entscheidung des Senats nicht zufrieden und ärgerte sich über Germanus wegen

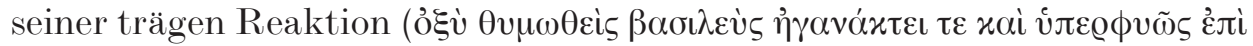

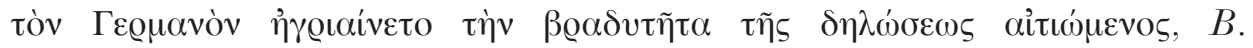
VII.32.47). Die näheren Ratgeber Justinians hetzten zwar den Kaiser gegen seinen Vetter Germanus auf (,,Sie bemühten sich immer darum, ihn mit fremdem Unglück zufriedenzustellen“, kommentiert verächtlich Prokop in B. VII.32.47), konnten ihre Meinung aber nicht durchsetzen. Was Prokop von dieser Clique um den Kaiser hält, hat er durch die Anklagen des Verschwörers Arsakes kurz davor angedeutet: Arsakes hat die engsten

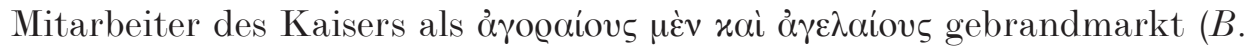
VII.32.17). Ein weiterer Topos der Kaiserkritik Prokops, der in den Anecdota hin und wieder auftaucht: Senat versus Emporkömmlinge, Tradition gegen Neuerung. Warum haben sich aber die homines novi des Kaisers jetzt nicht durchsetzen können? Verfügten sie etwa nicht über die Unterstützung des Kaisers?

${ }^{51}$ Nicht ohne Interesse ist die nicht erklärte Rückkehr von Vitalianus' Neffen Ioannes aus Italien zur Zeit der Verschwörung (cf. PLRE IIIA, s.v. Ioannes 46). Ioannes heiratete möglicherweise nach seiner Rückkehr Iustina, Germanus' Tochter, als die Kaiserin bereits tot war. Nach dem unerwarteten Tod von Germanus Ende 550 blieb sein Schwiegersohn viele Monate in Illyricum und leistete anscheinend widerwillig dem Kaiser Gehorsam. 
Geoffrey Greatrex hat meiner Meinung nach überzeugend gezeigt, daß man zu Justinians Zeiten kaum von einer einheitlichen senatorischen Oppositionsgruppe sprechen darf, da es damals viele „neue“ Mitglieder des Senats gab, die Justin und Justinian persönlich ernannt hatten ${ }^{52}$. Dennoch orientieren sich die Werke Prokops völlig nach einer traditionellen aristokratischen bzw. senatorischen Ideologie. Dieser anscheinende Widerspruch ist aber dann zu lösen, wenn wir annehmen, daß sich diese ,senatorische“ Opposition zum Teil nicht direkt gegen Justinian richtete, sondern gegen bestimmte Personen seiner unmittelbaren Umgebung. In diesem Sinne wäre eine mögliche Erklärung für diese sich immer mehr verzögernde Konspiration bzw. für die Schwäche des Kaisers in einem Geschehnis zu finden, das Prokop zwar nicht erwähnt, das aber sicherlich die unschlüssige Haltung der Verschwörer entschieden mitbestimmte. Es handelt sich um Theodoras Tod.

Wie oben erwähnt, war die Heiratspolitik Theodoras die Ursache der Verschwörung, da die Kaiserin diejenige war, die Artabanes an seiner Ehe mit Preiecta hinderte und somit seinen Zorn auslöste. Die Kaiserin aber starb im Juni 548, bevor Belisar in die Hauptstadt gelangte, wie Prokop in B. VII.30.4 schreibt. Dies bedeutet, daß die Verschwörung, die größtenteils gegen Theodora bzw. Theodoras Machtausübung gerichtet war, auf eine neue Basis gesetzt werden mußte. Nach Theodoras Tod öffneten sich langsam neue Perspektiven, die die Verschwörung vielleicht überflüssig machten und die die Verzögerungen der Verschwörer erklären können. Dies könnte die nach Theodoras Tod geschlossene Ehe zwischen Germanus und der ostgotischen Königin Matasuntha erklären. Die früher von Theodora verhinderte Ehe zwischen Johannes, Vitalianus' Neffe, und Justina, Germanus' Tochter, fand auch damals statt ${ }^{53}$. Prokop, der die Verschwörung ausführlich und sogar vom Standpunkt der Verschwörer beschreibt ${ }^{54}$ und

${ }^{52}$ Greatrex, Procopius the Outsider?

53 An. V.8 und PLRE IIIA, s.v. Ioannes 46.

${ }_{54}$ Dies bedeutet aber keinesfalls, daß Prokop an der Verschwörung direkt oder indirekt beteiligt war. Dennoch hat unser Historiker dieser Verschwörung große Bedeutung beigemessen und die großzügige Behandlung der Verschwörer seitens des Kaisers zweimal im Prooimion des De aed. (I.1.10 und 16) mit ausgesprochener Dankbarkeit erwähnt. Ob die kaiserliche Gnade gegenüber den Verschwörern die Meinung Prokops über Justinian zum Teil änderte und den Weg zum kaiserlichen Enkomion des De aed. bereitete? (cf. diesbezüglich M. Angold, Procopius' portrait of Theodora, in: C. CoNstantinides et alii [Hg.], $\mathrm{II} \Lambda \mathrm{E} \Lambda \Lambda \mathrm{HN}$. Studies in Honour of Robert Browning. Venedig 1996, 21-34, bes. 23-24). In De aed. bezeichnet Prokop die begnadigten Verschwörer

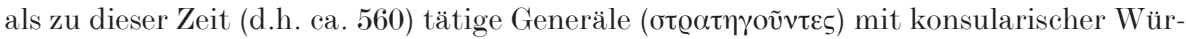
de. Normalerweise sieht man darin eine Anspielung auf die kaiserliche Gnade gegenüber Belisarios. Dennoch war Belisarios seit 550 (Abfassungszeit der Anecdota) für unseren 
der die Haltung von Artabanes und Germanus rechtfertigt, war sich sicherlich der neuen Lage bewußt.

Nachdem Theodora bereits gestorben war, war also ein direkter Putsch gegen Justinian nicht mehr nötig, weil es Mitglieder der eigenen Familie des Kaisers gab - wie Germanus, den Theodora von Machtstellungen fernhielt -, die jetzt einen neuen politischen Kurs einleiten und sogar den Thron in absehbarer Zeit besteigen konnten. Darüber hinaus war der Kaiser vom Tod seiner Frau sicherlich schwer getroffen, und seine Gesundheit war angegriffen, wie einige Quellen andeuten ${ }^{55}$. Er hatte damals das auch für heutige Begriffe hohe Alter von 65 Jahren erreicht. Es ist deswegen nicht auszuschließen, daß man sogar auf den bevorstehenden Tod des Kaisers wartete. Greatrex hat die Hoffnungen Prokops zu dieser Zeit trefflich geschildert ${ }^{56}$ :

Procopius can hardly have supposed that the emperor he so disliked would outlive him and reign for another twenty years: Justinian was already forty-five when he became sole emperor in 527. Of average build, he ate sparingly and slept little; in the 520s he suffered from a serious illness, and in the early 540 s he contracted the plague. The victim of an illnes and two serious plots in the 540 s alone seemed unlikely to remain on the throne for very much longer.

Historiker kein Held mehr, obwohl er immer noch gelegentlich im Dienst des Kaisers tätig war. Daher fragt man sich, ob an dieser Stelle des De aed. nicht eher Justinus, Germanus' Sohn, Konsul im Jahre 540, berühmter General bis zum Tode Justinians und sogar präsumptiver Nachfolger des Kaisers gemeint sein könnte. Justinus war auch irgendwie in die Verschwörung des Jahres $\mathbf{5 4 8}$ verwickelt, wie wir oben gesehen haben.

55 Die Niedergeschlagenheit und Schwäche des Kaisers in seinen letzten Jahren nach Theodoras Tod ist durch viele Quellen belegt (cf. Agathias IV.14; Corippus, In laudem Iustini II.265-276; Menandros Protector Fr. 5 [ed. Blockley, Liverpool 1985]). Laut Paulus Silentiarius, Descriptio Sanctae Sophiae 63-65, schwor Justinian häufig beim Namen der Kaiserin, als diese bereits tot war. Johannes von Ephesus (Commentarii de beatis orientalibus, ed. W. J. van Douwen und J. P. N. Land, Amsterdam 1889, 248) berichtet, daß der Kaiser den Wünschen seiner verstorbenen Frau immer noch Folge leisten wollte (rex, qua erat magna clementia, cum praesertim studeret ut voluntati uxoris etiam mortuae omnibus modis obtemperaret). Spätere Quellen bezeugen, daß Theodora die Politik ihres Mannes bestimmte (Zonaras XIV.6.1-9; Theophylaktos von Ochrida in seiner Apologie der Eunuchie, ed. P. Gautier, Thessalonike 1980

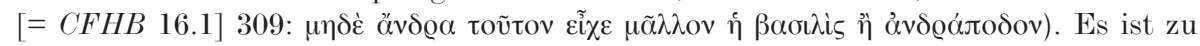
bezweifeln, daß diese späteren Quellen von der Geheimgeschichte abhängig sind, da bereits Johannes Lydus in seinem De magistratibus III.69 die Ohnmacht des Kaisers und die Entschlossenheit Theodoras schilderte, als es darum ging, Johannes den Kappadokier abzusetzen. Für einen Überblick über die Regierung Justinians ohne Theodora ef. J.W. Barker, Justinian and the Later Roman Empire, Madison 1966, 185-193 (,„Justinian without Theodora"). Für die eigentliche Macht Theodoras siehe weiter unten und A. 94.

${ }^{56}$ Greatrex, Procopius the Outsider? 218-219. 
Damit wir aber verstehen, warum Theodoras Tod einen politischen Wechsel hätte ermöglichen können, ist es nötig, hier die Rolle Theodoras in der justinianischen Regierung näher zu betrachten. Anschließend werden die Motive der Anecdota beleuchtet.

\section{Die Familie Theodoras}

Während Germanus' Persönlichkeit in den Anecdota kaum erwähnt und seine wichtige Rolle in Prokops Augen nur aus den letzten Kapiteln der Bella zu eruieren ist, ist die Figur Theodoras in den Anecdota zentral und taucht in den Bella nur gelegentlich auf. Daß der Tod Theodoras Prokop zur Abfassung der Anecdota veranlaßt hat, steht außer Zweifel. Der Kaiserin werden ganze Abschnitte des Werkes gewidmet, und sie teilt für Prokop mit dem Kaiser die Verantwortung für die meisten Entscheidungen. Ihre Macht erscheint unserem Historiker überwältigend, viele hohe Amtsträger wurden von ihr ernannt oder abgesetzt. Ihr Tod 548 bedeutete für Prokop sicherlich eine Hoffnung, die er jetzt in den neuen Ehebündnissen und in der geschwächten Stellung des Kaisers verwirklicht glaubte. So hat er 550 sein seit Jahren wachsendes Ressentiment gegen das kaiserliche Ehepaar in seinen Anecdota endlich offenbaren können. Mit Theodoras Tod waren also die Gefahren größtenteils geschwunden, die Prokop früher an solcher Kritik hinderten. Wir haben oben die Stelle am Anfang der Anecdota wiedergegeben, wo Prokop seine frühere Angst vor den „Trägern der Handlung" für geschwunden erklärt. Der Kaiser war zwar immer noch am Leben, er starb erst 15 Jahre später, stellte aber 550 anscheinend keine so große Gefahr wie früher dar. Ein Kurswechsel, mit oder ohne Justinian, schien damals vielen möglich. Hatte aber Theodora so große Macht, daß ihr Tod allein diesen Kurswechsel ermöglichte $?^{57}$

Theodora war in ihrer Jugend Schauspielerin in der anrüchigen Umgebung des konstantinopolitanischen Hippodroms, und hat mit aller Wahrscheinlichkeit Prostitution betrieben, obwohl wir allen übertriebenen Gerüchten bzw. Details, die Prokop über ihre „Karriere“ anhäuft, kaum Glauben schenken dürfen (cf. besonders $A n$. IX). Die Ehe zwischen dem römischen Senator Justinian, der damals als tatsächlicher Regent seines Onkels Justin agierte, und der unbekannten Prostituierten schien vielen ein Stein des Anstoßes. Grund war aber sowohl die niedrige Abstammung der Frau und ihre unehelichen Kinder ${ }^{58}$ als auch die hohe Position des Mannes.

${ }^{57}$ Über Theodora im allgemeinen siehe jetzt J. A. Evans, The empress Theodora. Partner of Justinian. Austin 2002.

${ }^{58}$ Sie hatte zumindest eine uneheliche Tochter und vielleicht auch einen Sohn, bevor sie Justinian heiratete. Der Sohn ist jedoch nur bei Prokop bezeugt (An. XVII.17-23). 
Ein ähnlicher Fall war die Heirat Justins. Die Frau Justins, Lupicina, ebenfalls niedriger Abstammung ${ }^{59}$, mußte vor ihrer Ankunft im Palast ihren Namen in den geeigneteren „Euphemia“ umändern, jedoch war ihre Heirat damit noch nicht akzeptabel, da Justin sie als Frau (Prokop: als Konkubine, $\pi \alpha \lambda \lambda \alpha x \eta$ ) gekauft hatte, als sie eine barbarische Sklavin war ${ }^{60}$. Justin mußte also seine Frau für von Geburt frei erklären, um sie heiraten zu können.

Ähnlich ging es bei der Heirat des Senators Justinian mit der Schauspielerin Theodora zu. Eine solche Heirat war gesetzlich verboten, da das schamlose Benehmen der Schauspielerinnen sie von einer regelrechten Heirat ausschloß. So sah sich Justin genötigt, ein eigenes Gesetz zu verabschieden, das allgemein die Schauspielerinnen zu einer gesetzlichen Heirat befähigte. In diesem Gesetz (Codex V.4.23) gab es nur zwei Vorbedingungen für eine solche Heirat: Erstens mußte die Frau ihre frühere Lebensweise aufgeben. Zweitens mußte der Kaiser jeden einzelnen Fall genehmigen. Mit der kaiserlichen Erlaubnis konnte die ehemalige Schauspielerin (scaenica) einen freien Mann heiraten, aber darüber hinaus war diesen Frauen auch jeder Makel (omnis macula) genommen, der mit ihrem früheren Leben verbunden war. Sogar ihre vorehelichen Töchter (filiae - nicht aber filii) waren legitimiert und somit zu gesetzlichen Ehen befähigt. Das Gesetz bezog sich auch auf den besonderen Fall der ehemaligen Schauspielerinnen, die bereits eine Würde (dignitas aliqua) vor ihrer Heirat bekommen hatten, und für die nicht einmal die kaiserliche Erlaubnis erforderlich war.

Zweifellos war dieser letzte Punkt konkret für die scaenica Theodora bestimmt, die bereits vor ihrer Ehe zur patricia ernannt wurde und sich damit die schändliche Demütigung einer speziellen petitio vor Kaiser Justin ersparen konnte. Mit dem Gesetz wurde auch der Tochter Theodoras die Tür zu einer legitimen Heirat geöffnet. Wir wissen tatsächlich, daß diese anonyme Tochter der Kaiserin einen Neffen des früheren Kaisers Anastasios heiratete. Die aus dieser Ehe hervorgegangenen drei Kinder, Anastasios - der bezeichnenderweise wie der frühere Kaiser hieß -, Athanasios und Johannes, verfügten über reichliches Vermögen und gingen auch vorteilhafte Ehen $\operatorname{ein}^{61}$. Dieses Gesetz legitimierte auch zweifellos die Heirat der

59 „Lupa“ ist eine durchaus übliche Bezeichnung für Prostituierte, ich bin aber nicht ganz sicher, daß der Name „Lupicina“ ursprünglich diese Bedeutung hatte, da die ethnische

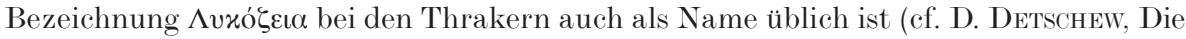
thrakischen Sprachreste. Öst. Akad. d. Wiss., phil.-hist. Kl., Schriften der Balkankommission, Linguist. Abt. 14, Wien 1976, 2. Auflage, 277).

${ }^{60}$ An. VI.17 und IX.49.

${ }^{61}$ L. Garland, Theodora, Wife of Justinian, in: Eadem, Byzantine empresses. Women and Power in Byzantium AD 527-1204. London-New York 1999, 37; Al. Cameron, The House of Anastasius. GRBS 19 (1978) 259-276. 
Schwestern von Theodora, da Sophia, eine Nichte von Theodora, Justinians Neffen Justinus, den künftigen Justinus II., heiratete. Mit diesem Gesetz bekräftigte also Theodora die Stellung ihrer Verwandten und bildete um sich eine Machtgruppe, deren sie dringend bedurfte, um ihre eigene Politik durchsetzen zu können.

Dazu stützte sich die Kaiserin meistens auf alte Freunde. Das bekannteste Beispiel ist die „patricia“" Antonina, die engste Freundin der Kaiserin, eine ehemalige Hetäre. Theodora hat sie mit dem berühmten General Belisar verheiratet, weil dessen Reichtum eine willkommene finanzielle Hilfe für die Kaiserin bedeutete. Dies war sicherlich der Grund dafür, daß Theodora ihrer Freundin Antonina die Heirat von Belisars Tochter Joannina mit ihrem Enkel Anastasios aufzwang ${ }^{62}$. Die Kontrolle, die Antonina über Belisar ausübte, wird von Prokop in den Anecdota dramatisch geschildert, und ist durch eine unabhängige westliche Quelle bestätigt, den sogenannten Liber Pontificalis. Dort wird uns erzählt, wie Antonina persönlich die Anklage gegen Papst Silverius wegen Verrats erhob. Die Szene findet in einem Saal des Quartiers der byzantinischen Truppen in Rom, im Hadriansmausoleum, statt: Antonina lehnt auf einem Ruhebett, während ihr stummer Ehemann zu ihren Füßen sitzt; der Papst wird von Antonina des Verrats angeklagt und sofort abgesetzt ${ }^{63}$. Antonina war aber keineswegs die einzige von der Kaiserin geförderte Freundin. Prokop kennt auch in $A n$. XVII.34-35 ältere Tänzerinnen und Hetären, die im Palast wohnten und sich um staatliche Angelegenheiten kümmerten. Obwohl wir Prokop eine gewisse Übertreibung zugestehen, bleibt es trotzdem Tatsache, daß die Macht der adligen Familien des Reiches mit Theodora (und Justinian) ziemlich zurückging. Laut Garland „Theodora's family - despite their lowly background and dubious origin - must have formed a distinctive group in the higher echelons of society in the capital" 64 .

${ }^{62}$ An. IV.37 und V.18-22. Die neue Deutung der Mosaiken Ravennas durch I. AndreescuTreadgold - W. Treadgold, Procopius and the Imperial Panels of S. Vitale. Art Bulletin 79 (1997) 708-723, verdeutlicht die außerordentliche politische Relevanz der Heirat zwischen Anastasios und Antonina: Laut den Verfassern sind nämlich beide jungen Ehepartner in den Mosaiken neben Antonina und Belisarios dargestellt worden.

${ }^{63}$ Liber pontificalis 6-7: Quo ingresso Silverio cum Vigilio soli in musileo, Antonina patricia iacebat in lecto et Vilisarius patricius sedebat ad pedes eius. Et dum eum vidisset Antonina, dixit ad eum: 'Dic, domne Silveri papa, quid fecimus tibi et Romanis, ut tu vellis in manus Gothorum tradere?' Adhuc ea loquente, ingressus Iohannis, subdiaconus regionarius primae regionis, tulit pallium de collo eius et duxit in cubiculum; expolians eum induit eum vestem monachicam et abscondit eum.

${ }^{64}$ Garland, Theodora 38. 
Um die Fortdauer der Macht ihrer Verwandten und Freunde auch nach ihrem eigenen Tod zu sichern, stand aber Theodora nur ein Mittel zur Verfügung: ein legitimer Sohn aus ihrer Ehe mit Justinian. Der erwünschte Sohn kam aber nie, ohne daß wir über die Gründe informiert sind ${ }^{65}$. Daß der Kaiserin die Geburt eines Sohnes am Herzen lag, wird von einer zeitgenössischen Quelle bestätigt, der Vita des Heiligen Sabas, die dessen Schüler Kyrillos von Skythopolis (ca 524-568/9) schrieb. An einer Stelle dieses Werkes ${ }^{66}$ besucht Sabas die Kaiserin in ihren Gemächern. Theodora bittet den heiligen Mönch zweimal um seinen Segen, damit ihr ein Sohn geboren werde. Der orthodoxe Sabas lehnt aber wiederholt die Bitte der monophysitischen Kaiserin ab und verläßt das Zimmer. Kyrillos schreibt, daß Theodora traurig wurde, weil Sabas ihrer Bitte nicht zustimmen woll-

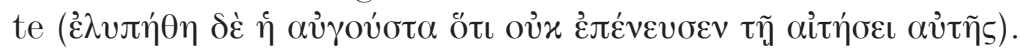

Die Gründe, die Sabas angibt, um sich dem Wunsch der Kaiserin zu widersetzen, sind religiöser Natur: er wolle nicht, daß der nachgeborene Sohn (als künftiger Kaiser der Römer) aus der Doktrin der (monophysitischen) Severianer sauge und wie zur Zeit des Anastasios Unruhen in der Kirche stifte. Dennoch gab es wahrscheinlich in der Hauptstadt auch Kreise, denen die Macht Theodoras nicht aus religiösen, sondern bloß aus gesellschaftlichen Gründen zuwider war, und die sich deswegen davor fürchteten, daß Justinians Nachfolge von ihr bestimmt würde. Als Justinian 542 an der Pest erkrankte, entstanden bereits Gerüchte im römischen Heer über die Nachfolge. Prokop, der uns die Episode überliefert (An. IV.1-12), sagt nur, daß die Kommandanten einen ,zweiten“ Justinian fürchteten, fügt aber hinzu, daß sich Theodora von diesen Gerüchten persönlich getroffen fühlte. Ob die Kaiserin damals an eine Nachfolge innerhalb ihrer eigenen Familie dachte, wissen wir nicht ${ }^{67}$. Dennoch ist die von Prokop bezeugte Einmischung der Kaiserin in die Heiratspläne vieler adeliger Familien der

${ }^{65}$ Hatte das mit der schlechten Gesundheit der Kaiserin zu tun? Laut Victor Tonnennensis s.a. 549 starb die Kaiserin an Krebs: Theodora Augusta Calchedonensis synodi inimica canceris plaga corpore toto perfusa vitam prodigiose finivit. GaRLand, Theodora, setzt die Krankheit der Kaiserin in Beziehung mit ihren häufigen Besuchen der Bäder, die Prokop in An. XV.7 erwähnt. Ihre wiederholten Abtreibungen (An. IX.19) könnten ebenso ihrer Gesundheit geschadet haben. Eine Sterilität des Kaisers ist selbstverständlich auch nicht auszuschließen. Interessant ist es aber hier, daß Victor Tonnennensis Theodoras Tod exemplarisch schildert, etwa wie die Strafe einer häretischen Kaiserin, was auch Prokops Absicht gewesen sein könnte.

${ }^{66}$ Kyrillos von Skythopolis, Leben des heiligen Sabas, ed. E. Schwartz, Leipzig 1939, $173-174$.

${ }^{67}$ Sophia, die Nichte Theodoras, hatte möglicherweise vor 542 den künftigen Kaiser Justin geheiratet, da Justin 565 bereits eine verheiratete Tochter hatte (cf. Av. Cameron, The empress Sophia, Byz 45 [1975], 5-21, hier 6). 
Hauptstadt wahrscheinlich als Schutz ihrer eigenen Interessen zu verstehen. So treibt Theodora zum Beispiel zwei Schwestern aus einer vornehmen Familie (mit drei Generationen von Konsulen!) zu einer Heirat mit zwei Männern niedriger Abstammung, und zwar nachdem sie beide zu Witwen geworden waren ${ }^{68}$. Es ist offensichtlich, daß Theodora sich vor einer zweiten Ehe dieser Schwestern fürchtete, da eine solche Ehe potentiell ihren Plänen entgegenstehen hätte können. Wenn Prokop die Namen der Schwestern nicht aus Respekt verschwiegen hätte ${ }^{69}$, könnten wir vielleicht ihre Gründe näher bestimmen. Es ist aber nicht nötig, weitere Einzelfälle aus den $A n$ ecdota anzuführen. Es reicht uns hier eine Aussage Prokops (An. XVII.28-

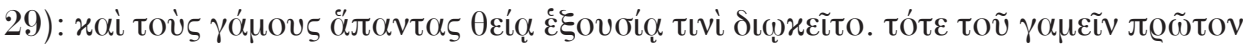

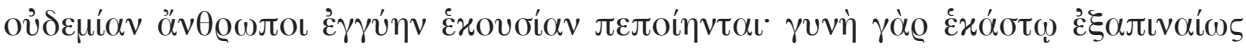

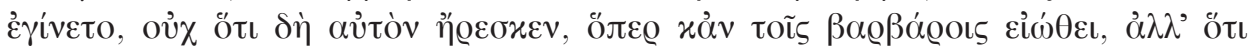

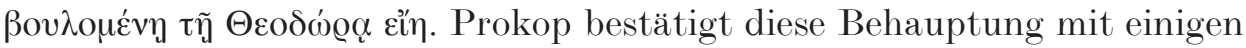
Fällen, in denen die Kaiserin bereits arrangierte Ehen stört. Es ist zwar möglich, daß die Kaiserin gelegentlich als Schützerin der Frauenrechte hervortrat, da sie von einigen Gelehrten als Anregerin der fortschrittlichen Gesetzgebung Justinians zu Gunsten der Frauen angesehen wird ${ }^{70}$. Möglich ist es auch, daß diese aktive Rolle Theodoras gegenüber den Frauen Prokop besonders geärgert hat. Dennoch hat man allgemein den Eindruck, daß Theodora öfters dabei in eigenem Interesse agiert. Dies war sicherlich der Fall bei der oben erwähnten Ehe des Germanus, die nur nach Theodoras Tod stattfinden konnte. Wenn die Verschwörer des Jahres 548 meinten, keiner der Verwandten Justinians werde sich ihren Plänen widersetzen ( $B$. VII.32.10), müssen wir hier vielleicht zwischen den Zeilen lesen und darunter verstehen, daß die Familie der Kaiserin die eigentliche Gefahr war? ${ }^{71}$

Germanus war für die Kaiserin noch gefährlicher als andere Mitglieder der Familie Justinians, ein Umstand, der den heftigen Haß der Kaiserin gegen ihn $\left(A n\right.$. V.8) verständlich macht ${ }^{72}$. Er war bereits 519 ,,vir illustris“ und hatte in den darauffolgenden Jahren wichtige Posten und Ehren be-

${ }^{68}$ An. XVII.7-15. Die von C. Foss, The empress Theodora. Byz 72 (2002) 141-176 vorgelegte Identifikation dieser zwei Männer (S. 161-162) ist m. E. nicht überzeugend.

${ }^{69}$ In $A n$. XV.25 verschweigt Prokop ebenfalls den Namen eines Patrikios, der von der Kaiserin beleidigt wurde, um seine Demütigung nicht zu verewigen. Es ist anzunehmen, daß ähnliche Gründe Prokop veranlaßt haben, viele Namen zu verschweigen.

${ }^{70}$ Cf. diesbezüglich J. E. Sprutт, L’influence de Théodora sur la législation de Justinien. Revue internationale des droits de l'Antiquité 24 (1977) 389-421, bes. 402.

${ }^{71}$ Petros Patrikios, bekannt als Agent Theodoras in Italien (s. unten in Abschnitt 5), verfaßte Abhandlungen über Kaiserkrönungen (heute erhalten im Zeremonienbuch), die vielleicht in Verbindung mit den Interessen Theodoras zu setzen sind.

72 Dieselbe Meinung vertritt Evans, Theodora 49-50 und 119. 
kommen, obwohl er erst nach dem Tode der Kaiserin als vermeintlicher Nachfolger Justinians hervortritt ${ }^{73}$. Dennoch gab es vielleicht noch etwas in der Familie von Germanus, das die Kaiserin besonders beunruhigte. Eine Stelle in der Geschichte der Goten von Jordanes, die bis jetzt von der Kritik kaum in diesem Zusammenhang beachtet wurde, kann uns hierbei behilflich sein.

\section{Germanus und Matasuntha}

Anfang 551 schrieb der Gote Jordanes in Konstantinopel in lateinischer Sprache seine Getica, oder Geschichte der Goten, die er als Pendant zu seinen gleichfalls zu dieser Zeit redigierten Romana konzipierte ${ }^{74}$. Obwohl Jordanes in seinen Getica mehrmals Germanus erwähnt, interessiert uns hier nur eine kleine Stelle kurz vor Ende des Werkes (Getica 314). Dort wird von der Ehe zwischen Matasuntha, Enkelin Theoderichs, und Germanus folgendes gesagt: Mathesuentam vero iugalem eius fratri suo Germano patricio coniunxit imperator. De quibus posthumus [v.l. post humatum] patris Germani natus est filius idem Germanus, in quo coniunctum [v.l. coniuncta] Aniciorum genus [v.l. gens] cum Amala stirpe, spem adhuc utriusque generis domino praestante promittit. Mit diesen Sätzen begrüßt Jordanes den 551 posthum geborenen Sohn des Germanus und der gotischen Königin Matasuntha als künftige Hoffnung (spes) der Familien der Amaler und Anicier. Da mit den Amalern das ostgotische Königshaus gemeint ist, zählt Jordanes den General Germanus zu den Aniciern.

Die Anicii waren damals eines der vornehmsten Geschlechter im Reich. Anicius Olybrius war 472 weströmischer Kaiser und seine Tochter Anicia Juliana hatte nicht nur die größte Kirche des Reiches, die Polyeuktos Kirche, gebaut und so Justinian zum Bau der Hagia Sophia angeregt ${ }^{75}$, son-

${ }^{73}$ Für die Karriere von Germanus of. PLRE s.v. Germanus 4, 504-507.

${ }^{74}$ Die Abfassungszeit beider Werke ist umstritten. Ich bin mit B. Croke, Cassiodorus and the Getica of Jordanes, Classical Philology 82 (1987) 117-134 (hier A. 1 und 121-122) und P.J. Heather, Goths and Romans 332-489. Oxford 1991, 47-49, in der früheren Datierung der Getica vor 31. März 551 (Ende des 24. Regierungsjahres Justinians, auf das Jordanes hinweist) einverstanden. Die Romana wurden vielleicht ein bißchen später als die Getica, etwa im Winter 551-552, abgefaßt. Heather hat die von W. Goffart, The narrators of barbarian history (A.D. 500-800). Princeton 1988, vorgeschlagene Datierung ca. 554 überzeugend widerlegt. Der Unterschied zwischen Abfassungszeit und Ausgabe beider Werke kann die gelegentliche Erwähnung späterer Ereignisse erklären.

${ }^{75}$ Für die Ausgrabungen ef. R.M. Harrison, A Temple for Byzantium. London 1989, bes. 137-144. Siehe auch P. Speck, Juliana Anicia, Konstantin der Große und die Polyeuktoskirche in Konstantinopel, Poikila Byzantina 11 (1991) 133-147, und besonders J. 
dern auch als Mäzenin die berühmte Dioskurides Handschrift (heute in Wien) in Auftrag gegeben. Unter den Nachkommen der Anicia Juliana sind uns im Laufe des 6 . Jahrhunderts viele weitere Anicier bekannt, die gelegentlich auch wichtige Posten innehatten ${ }^{76}$. Die potentielle Gefahr, die diese Familie für Emporkömmlinge wie Justinian und Theodora bedeutete, war vielleicht nur durch eine Verbindung beider Geschlechter aufzuheben. So wäre eine mögliche Ehe zwischen Proba, Ururenkelin Anicia Julianas, und einem Verwandten Theodoras namens Georgius zu erklären ${ }^{77}$. Was für eine Verbindung hatte aber Germanus zu den Aniciern? Er war, wie bereits gesagt, ein Vetter Justinians, so daß seine vermeintliche Zugehörigkeit zu den Aniciern nur bedeuten kann, daß ein Bruder bzw. eine Schwester Kaiser Justins I. eine Ehe mit einem Familienmitglied der Anicier geschlossen hatte, aus der Germanus hervorging ${ }^{78}$. Wir wissen leider nicht, wer die Eltern von Germanus waren. Von den zwei Brüdern des Germanus hatte der eine, Justus, auch einen lateinischen Namen, der andere aber hieß Boraides, eine Benennung, die auf einen illyrischen Ursprung hindeutet ${ }^{79}$. Hatte Boraides seinen illyrischen Taufnamen beibehalten, während sich Justus und Germanus dagegen einen angemesseneren lateinischen Namen zu eigen gemacht hatten ${ }^{80}$ ? Oder ist der Namensunterschied zwischen den

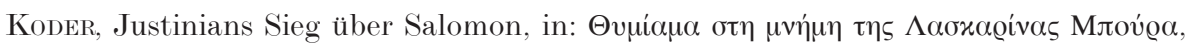
Athen 1994, 135-142.

${ }^{76}$ Ein Stammbaum der Familie ist in Stemma 3 des PLRE II, 1309 zu sehen. Cf. auch A. Momigliano, Gli Anicii e la storiografia latina del VI sec. D.C., in: ID., Secondo contributo alla storia degli studi classici, Roma 1960, 231-253.

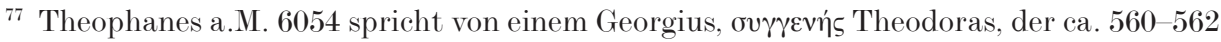
curator divinae domus Marinae war und anscheinend gegen Justinian konspirierte. Im Artikel „Georgius 7“, des PLRE IIIA (515), wird dieser Georgius mit dem Mann Probas identifiziert.

${ }^{78}$ Im Index zu seiner Ausgabe von Jordanes schreibt Mommsen folgendes zur in Getica 314 erwähnten Verbindung der Aniciorum gens mit Germanus: „Germani, Iustiniani fratris filii, parentum cum nomina ignorentur, fieri potest, ut mater Anicia fuerit vel ad Anicios aliqua ratione pertinuerit, filia fortasse Aniciae Iulianae, quam genuerunt imperator Occidentis Olybrius et Placidia filia Valentiniani III“. Momigliano, Gli Anicii 239, schreibt seinerseits: „Il modo più semplice per spiegare l'allusione alla gente degli Anicii in questo contesto è di supporre che Germanus padre di Germanus fosse figlio di una donna Anicia. In tal caso il padre di Germanus avrebbe sposato una Anicia intorno al 505 d. Cr.; e questa Anicia potrebbe essere una figlia di Anicia Iuliana."

${ }^{79} \mathrm{Zu}$ dem bei den Thraker üblichen Namen Boraides ef. Detschew, Die thrakischen Sprachreste, 80, der den Namen in Verbindung mit albanischen Wörtern setzt.

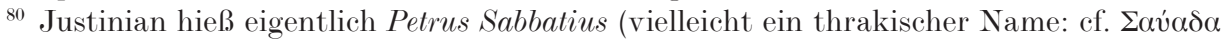
in Detschew, Die thrakischen Sprachreste, 427), nahm aber später den Namen Iustinianus zur Ehre seines Onkels Justinus an. Es ist anzunehmen, daß Justinus nicht der Taufname des Kaisers war, so wie seine Frau Euphemia ursprünglich Lupicina hieß. 
Brüdern eher auf eine ungleiche Ehe zwischen einem Illyrer und einer römischen Anicia zurückzuführen? Wie dem auch sei, es gibt keinen Grund, um die von Jordanes postulierte Verbindung von Germanus mit den Aniciern zu bezweifeln ${ }^{81}$.

Die außerordentliche Bedeutung der Ehe der Amalerin Matasuntha mit dem „Anicier“ Germanus war sicherlich allen Zeitgenossen klar ${ }^{82}$. Der in Konstantinopel ansässige Gote Jordanes widmete sich also den Getica nicht aus reiner Neugier bzw. aus Interesse an der Geschichte seines Volkes ${ }^{83}$, sondern möglicherweise weil er mit diesem Werk irgendwie zur erhofften Annäherung beider Reiche beitragen wollte ${ }^{84}$. Die schwankende ostgotische Politik Justinians wurde sicherlich nicht von den vielen italienischen Adligen gebilligt, deren Anwesenheit im damaligen Konstantinopel, sozusagen als Flüchtlinge aus dem Kriegsschauplatz, genügend belegt ist ${ }^{85}$. Der vornehmste von ihnen war vielleicht, abgesehen von Papst Vigilius, Flavius Rufus Petronius Nicomachus Cethegus, wahrscheinlich Angehöriger der Anicier ${ }^{86}$. Prokop berichtet in Bella VII.35.9-11, wie dieser vergebens Druck auf Justinian ausübte, um ihn zu einer energischen Intervention in Italien zu bewegen: Der Kaiser kümmerte sich aber inzwischen, so Prokop, nur um religiöse Angelegenheiten. Diese verlangte militärische Intervention schloß aber nicht politische Maßnahmen aus, mit denen man, wie Brian

In diesem Kontext kann der Name Iustus nur als eine typisch "römische" Bezeichnung betrachtet werden.

${ }^{81}$ Jordanes in Getica 81 und 251 bezeichnet Germanus richtig als fratruelis Justinians, während die erwähnte Stelle der Getica 314 von einem fratri Justinians spricht. Der Herausgeber Mommsen schlägt eine Verbesserung in fratueli vor, was angesichts des verdorbenen Zustandes des Textes durchaus annehmbar wäre. Im Lateinischen wird aber auch häufig frater mit der Bedeutung von „Vetter“ gebraucht, so daß die Verbesserung in fratrueli nicht notwendig scheint. Die Zuverlässigkeit der Aussage von Jordanes wird daher wegen dieses kleinen textkritischen Problemes nicht gemindert.

${ }^{82}$ Viele Quellen behaupten, die frühere Ehe Matasunthas mit König Vitiges sei erzwungen gewesen. Vielleicht hatte diese Notiz mit der offiziellen Propaganda zu tun, die nach ihrer Heirat mit Germanus entstanden war (cf. B. V.11.27; Jordanes, Romana 373; Marcellinus 105).

${ }^{83}$ So Heather, Goths and Romans 49-50.

${ }^{84}$ Jordanes benutzt als Quelle für die Getica die Geschichte der Goten von Kassiodor, der lange Jahre als Kanzler der Ostgoten gewirkt und seine zwölf Bücher De origine actibusque Gothorum etwa zwanzig Jahre vor Jordanes geschrieben hatte. Ich bin mit Goffart, The narrators, und Heather, Goths and Romans, darin einig, daß Jordanes kein bloßer Kopist des verlorenen Werkes von Kassiodor war, wie es früher von Momigliano angenommen wurde. Das Werk Kassiodors war sicherlich nicht weit verbreitet und wurde Jordanes vielleicht durch persönliche Kontakte übermittelt.

85 Momigliano, Gli Anicii.

${ }^{86}$ Momigliano, Gli Anicii 234, 239-240. 
Croke zurecht betont hat ${ }^{87}$, die Einheit der ostgotischen Truppen untergraben wollte, und dazu, so meine ich, eine Basis für die Versöhnung zwischen Ostgoten und Römern nach dem zu erwartenden Sieg der kaiserlichen Truppen etablieren hätte können. Der „Anicier“ Germanus spielte 550 die entscheidende Rolle in diesen Plänen. Es ist in dieser Hinsicht kein Zufall, daß Jordanes den posthum geborenen Sohn von Germanus als spes beider Familien, d.h. beider Völker (Ostgoten und Römer), bezeichnet. Von Bedeutung ist auch, daß Jordanes etwa gleichzeitig an den Romana arbeitete, die ein Parallelwerk zu den Getica bildeten und die römische Geschichte knapp darstellen sollten. Jordanes konnte seine Arbeit unmittelbar nach Germanus' Heirat mit Matasuntha ca. 549 bereits begonnen haben. Die gelegentliche Kritik an Justinian, sowohl in den Getica als auch in den Romana, schließt die Möglichkeit aus, daß er einem offiziellen Auftrag Folge leistete $^{88}$. Wahrscheinlicher ist es, daß Jordanes, wie Prokop, einen Kurswechsel in der Regierung mit der möglichen baldigen Ernennung von Germanus zum Nachfolger Justinians erwartete und daß dieser Umstand ihn zur Abfassung seines Werkes veranlaßte ${ }^{89}$.

Dennoch waren Jordanes und Prokop gleichermaßen enttäuscht, als Ende 550 Germanus plötzlich starb. Prokop stellte seine Geheimgeschichte bereits 550 ein und publizierte Anfang 551 mit kleinen Ergänzungen seine Bella I-VII. Jordanes hatte möglicherweise das Ende der Romana und der Getica leicht retuschiert, als beide Werke 551/552 veröffentlicht wurden. Der posthum geborene Sohn von Germanus wird in diesen zwei Werken kurz vor dem Ende erwähnt ${ }^{90}$. Die Getica brechen sogar mit einer überraschenden laudatio Justinians ab, die unmittelbar auf die Erwähnung des posthumen Kindes des Germanus kommt. Diese Stelle sieht wie ein späterer Zusatz des Verfassers aus.

Es ist meiner Meinung nach klar, daß der Tod Theodoras nicht nur Germanus' Ehe mit Matasuntha ermöglichte, sondern auch ein neues politisches Klima einleitete, in dem die Werke des Jordanes und des Prokop ihren Platz finden konnten. Theodora hatte sich immer einer politischen Annäherung an Italien widersetzt, da diese Annäherung möglicherweise ein Ehebündnis zwischen den Amalern und einem Verwandten Justinians vor-

87 Croke, Cassiodorus 132.

88 Cf. Heather, Goths and Romans 43 für Jordanes' Kritik an Justinian. Die verhüllte Kritik an Justinian (z.B. wegen Nachlässigkeit in Getica 5.37) erinnert an ähnliche Aussagen Prokops gegen den Kaiser in seinen Bella. Cf. auch Croke, Cassiodorus 128.

${ }^{89}$ Für den Fortsetzer der Chronik des Marcellinus, der sein Werk wieder im Todesjahr Theodoras (548) abrupt enden ließ, ist Germanus ein nationaler Held. Noch ein Zufall? Cf. B. Croke, Count Marcellinus and his chronicle. Oxford 2001, 226.

${ }^{90}$ Mommsen, praef. XIV-XV. 
aussetzte, das ihre eigene Familie endgültig aus dem politischen Spiel verdrängt hätte. Dies war wahrscheinlich der Grund, der Theodora zur Ermordung Amalasuntas, der damaligen Königin der Ostgoten, bewog, eine Aktion, die 535 zum casus belli für den byzantinischen Angriff geworden war. Prokop erzählt in An. XVI, wie mißtrauisch die Kaiserin der adligen Abstammung, Schönheit und Entschlossenheit der Königin Amalasunta gegenüberstand. Theodora traute auch ihrem Mann Justinian nicht, vor

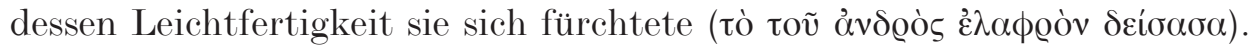
Da Amalasunta von König Theodahat wegen ihrer prorömischen Haltung bereits eingekerkert worden war, versuchte Theodora laut Prokop, Theodahat zur Ermordung der Königin zu drängen. Dies habe sie schließlich durch die Vermittlung des kaiserlichen Gesandten Petros Patrikios erreicht, der 534/5 den ostgotischen Hof besuchte. Ein Brief Theodahats an Theodora scheint diese Version Prokops zu bestätigen ${ }^{91}$. Theodora hatte aber nicht aus Eifersucht gehandelt, wie Prokops Erzählung andeutet. Eine Ehe Justinians mit Amalasunta ist undenkbar, da der christliche Kaiser, den die Romanisten als „legislator uxorius“ zu bezeichnen pflegen, zuerst seine Frau gegen seine eigenen Gesetze hätte verstoßen müssen ${ }^{92}$. Demgegenüber ist die Furcht Theodoras vor einem Ehebündnis zwischen Amalasunta und einem Verwandten Justinians wohl ein möglicher Grund für ihre Einmischung in die äußere Politik des Kaisers in Italien. Erst nach dem

${ }^{91}$ Der Brief ist in den Varia Kassiodors erhalten (10.20). Dort schreibt Theodahat an die Kaiserin unter anderem folgendes: Nam et de illa persona de qua ad nos aliquid verbo titillante pervenit, hoc ordinatum esse cognoscite, quod vestris credidimus animis convenire. Diese Worte werden normalerweise als eine versteckte Anspielung auf Theodoras Befehle betreffend das Schicksal der Amalasuntha verstanden. In der Sammlung Kassiodors sind noch ein zweiter Brief des Königs (10.23) und zwei weitere seiner Frau Gudeliva (10.21 und 10.24) an Theodora erhalten. B. Rubin, Das Zeitalter Justinians. Bd. II (C. Capizzi Hg.). Berlin 1995, 80-90, der die ganze Angelegenheit ausführlich studiert und die Korrespondenz zwischen Ravenna und Theodora übersetzt, ist von der Verwicklung Theodoras in den Tod Amalasunthas völlig überzeugt. Dagegen aber V.A. Sirago, Amalasunta. La regina (ca. 495-535). Milano 1998, 94-95. Evans, Theodora 65 glaubt, daß Theodahat in seinem Brief mit illa persona nicht auf Amalasuntha, sondern auf den neuen Papst Agapetus anspielt, der am 13. Mai 535 gewählt wurde, während Amalasuntha hingerichtet wurde. Dennoch wird Agapetus einige Zeilen weiter oben in demselben Brief expressis verbis erwähnt und zwar in einem anderen Kontext. Ich bin nicht überzeugt, daß Theodora hier einfach als Agentin Justinians agierte, wie Foss, Theodora 162-163 und 172 meint.

${ }^{92}$ Es gab anscheinend niemals ernste Zwiste zwischen Justinian und Theodora, obwohl die Entscheidungen der Kaiserin gelegentlich Justinians Maßnahmen widersprachen. Prokop weiß nur von Sexskandalen der Theodora vor ihrer Heirat mit Justinian (die Ausnahme ist $A n$. XVI.11, die im Grunde genommen eher für Theodoras Treue spricht). 
Tode Theodoras war wieder eine Annäherung zwischen Justinian und den Amalern durch die Heirat von Germanus mit Matasuntha möglich ${ }^{93}$.

\section{ZuSAMMENFASSUNG}

Als Theodora 548 starb, erhofften sich viele adelige Familien in Konstantinopel einen Kurswechsel in der Regierung Justinians. Die Kaiserin hatte bis jetzt entschieden die Politik des Reiches bestimmt, teils wegen ihrer religiösen Überzeugung, teils um die Teilnahme ihrer Familie an der Macht zu sichern ${ }^{94}$. Nach ihrem Tod wurde eine gegen den Kaiser bereits in Gang gesetzte Verschwörung, in die Germanus, Justinians Vetter, irgendwie verwickelt war, gestoppt. Kaiser Justinian, dessen Gesundheit seit 542 anscheinend angeschlagen war, blieb eine gewisse Zeit untätig. Der Senat gewann sogar die Oberhand, indem er dem Kaiser die Begnadigung der ertappten Verschwörer aufzwang. Eheschließungen, die lange Zeit von Theodora verhindert worden waren, fanden jetzt ungehindert statt. So konnte Germanus ca. 549 die Ostgotin Matasuntha, Enkelin Theoderichs des Großen, heiraten. Auch die Tochter des Germanus, Justina, heiratete zu dieser Zeit Ioannes, den Neffen des berühmten Befehlshabers Vitalianus. Germanus, der mit seiner Heirat breitere Schichten der ostgotischen Mächtigen für das Reich gewinnen konnte, wurde das Kommando eines bevorstehenden Feldzuges nach Italien anvertraut. Das Ende des bereits 15 Jahre währenden italischen Krieges schien damals in greifbarer Nähe zu liegen. Jordanes, ein Gote, begann zu dieser Zeit in Konstantinopel, eine lateinische Doppelgeschichte der Römer und Goten zu schreiben, die propagandistisch gesinnt war und wahrscheinlich mit dem triumphalen Ein-

93 Cf. Evans, Theodora 106.

${ }_{94}$ Was die eigentliche Macht Theodoras in der Regierung betrifft, herrscht heute Uneinigkeit. C. Pazdernik, Our Most Pious Consort, Classical Antiquity 13 (1994) 256-281 glaubt, daß Theodora kaum ihre eigenen Entscheidungen durchsetzen konnte, seine Argumente sind aber meiner Meinung nach nicht schlüssig. Für Theodoras Macht sprechen verschiedene Tatsachen, wie z.B. daß Theodora eigenständig mit ausländischen Gesandten verhandelte, daß sie Beamte absetzte, die sich ihren Wünschen widersetzten, daß sie über Vertrauensmänner in verschiedenen Gebieten des Reiches verfügte (z.B. in Ägypten oder Italien), daß sie Patriarchen ernennen und Päpste absetzen konnte. Evans, Theodora passim glaubt an ein politisches Spiel des kaiserlichen Ehepaares, besonders in den religiösen Streitigkeiten zwischen Monophysiten und Chalkedonensern. Er zitiert aber auch Fälle, wo Theodora anscheinend ohne Zustimmung ihres Mannes agierte, wie z.B. bei der Ernennung des ersten monophysitischen Bischofs und Gründers der syrischen Kirche, Jakob Baradeus (S. 94-97). Ist es ein Zufall, daß Justinian genau zu dieser Zeit an der Pest erkrankt war? Cf. auch L. JAMes, Empresses and Power in Early Byzantium. Leicester 2001, 86-87. 
zug von Germanus in Italien schließen sollte. Germanus, Angehöriger des mächtigen Geschlechts der Anicier (mütterlicherseits?) und Vetter Justinians, war zweifellos 550 der beste Nachfolger für den Thron.

Die lange Regierung Justinians näherte sich anscheinend in den Augen vieler Zeitgenossen einem vorzeitigen Ende. Prokop, der lange Jahre an einer Geschichte der Kriege Justinians gearbeitet hatte, dachte 550 an einen Anhang zu seinen Kriegen bzw. Bella, in dem er endlich über die von ihm in den früheren Bänden verschwiegenen Übeltaten des kaiserlichen Ehepaares ohne Furcht sprechen konnte. Diese Anecdota Prokops, ohne besondere Einleitung als Schlußteil der Bella gedacht, wurden mit aller Wahrscheinlichkeit konzipiert, um den Kurswechsel von Germanus' Regierung einzuleiten. Daß ein neuer Kaiser durch die Mängel seines Vorgängers positiv dargestellt wird, ist ein durchaus übliches Verfahren in der byzantinischen Geschichte bzw. Geschichtsschreibung, von dem wir über genügend Beispiele verfügen. Es reicht hier, die ikonodulische Propaganda gegen die Ikonoklasten oder die makedonische gegen die Amorier zu nennen. Prokop, der seine Hoffnungen mit einer unbestimmten Oppositionsgruppe teilte $^{95}$, dachte also möglicherweise an eine neue von Germanus begründete Dynastie, die sich ideologisch von der Familie Justinians unterscheiden würde.

Weder Prokop noch Jordanes hatten mit dem plötzlichen Tod von Germanus vor dem Winter 550-551 gerechnet ${ }^{96}$. Jordanes publizierte trotzdem 552 seine Romana und Getica mit kleinen Ergänzungen im Schlußteil, obwohl beide Werke möglicherweise 551 bereits fertig waren. Genau dasselbe tat Prokop mit seinen Bella I-VII Anfang 551. Am Ende des VII. Buches erschien sogar eine kurze aber bedeutungsvolle Laudatio des Germanus, während Justinian an keiner Stelle der Bella ein ähnliches Lob zugekommen war. Dagegen wurden die als VIII. Band des Werkes Prokops konzipierten Anecdota nicht veröffentlicht, erstens weil die von Prokop erwartete politische Wende vorläufig ausgeschlossen war, und zweitens weil Justinian, der in diesem letzten Band heftig angegriffen wurde, immer noch das Reich regierte. So wurde der VIII. Band zu unseren heutigen Anecdota, ein unediertes Werk, das in Prokops Schreibtischlade steckte.

Das Werk blieb also unediert, ist aber in seinem heutigen Zustand nicht weit von der Vollendung entfernt. Die Episoden reihen sich zwar ohne chronologische Folge aneinander, manche Punkte werden öfters wieder-

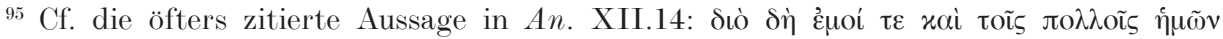

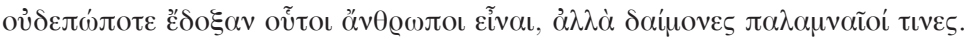

${ }_{96} \mathrm{Zu}$ dieser Zeit starb auch Johannes, der die Nichte Justinians geheiratet hatte. 
holt, es fehlen auch Berichte über die Todesumstände von Justinian und Theodora ${ }^{97}$, dennoch stehen wir vor keinem bloßen Dossier, sondern vor einem Werk, das vielleicht eine letzte Hand bzw. eine allgemeine Durchsicht benötigte, bevor es zum Kopisten geschickt wurde. Die Anecdota wurden aber nie publiziert, sie wurden zum ersten Mal im Lexikon Suda im 10. Jh. kurz erwähnt und später von byzantinischen Autoren kaum benutzt. Es ist also nicht verwunderlich, daß die Anecdota bestimmte Unregelmäßigkeiten aufweisen.

Prokop veröffentlichte 553 das VIII. Buch der Bella mit einer gesonderten Einleitung, in der er auschließlich über die neue einheitliche chronologische Darstellung der Ereignisse nach der Publikation von Bella I-VII schrieb. Es ist anzunehmen, daß Prokop damals auf eine künftige Publikation der Anecdota bereits verzichtet hatte. Wir wissen aber nicht, wie er eigentlich die politische Entwicklung seit Germanus' Tod betrachtete. Vielleicht war die alleinige Herrschaft Justinians für Prokop langsam erträglicher geworden, obwohl die indirekte Kritik gegen den Kaiser auch in Bella VIII geübt wird.

Eine gewisse Annäherung an den Kaiser sollte aber erst in den nächsten Jahren stattfinden, da es ansonsten unerklärlich ist, wie Justinian unseren Historiker mit der Abfassung eines Panegyrikus wie De aedificiis beauftragt haben sollte, besonders wenn man in Betracht zieht, daß die indirekte Kaiserkritik der Bella von Justinian sicherlich nicht übersehen werden konnte. Das Werk De aedificiis wurde von Prokop ab der Mitte der fünfziger Jahre redigiert und rühmt wiederholt den Kaiser für seine stete Bautätigkeit $^{98}$. Diese Laudatio Justinians hat aber Prokop, wenn nicht routinemäBig, so doch größtenteils anhand offizieller Quellen durchgeführt. Das Werk blieb sogar unvollendet, wie Prokop selbst am Ende zugibt ${ }^{99}$. Man könnte also eher von einer äußeren Versöhnung des Historikers mit dem Kaiser sprechen. Wie dem auch sei, das Erscheinen von De aedificiis bedeutete den endgültigen Verzicht auf eine künftige Publikation der Anecdota. Prokop dachte zu dieser Zeit wahrscheinlich nicht mehr an die seit Jahren nicht revidierten und größtenteils überholten Anecdota, während er im Gegenteil

97 Die Darstellung der Umstände, unter denen das kaiserliche Ehepaar starb, gehörte möglicherweise zum einleitenden Abschnitt der neuen Regierung des Germanus.

98 Ich bin der Ansicht, daß sich Prokop aus irgendwelchen Gründen zur Abfassung dieses Werkes genötigt sah, denke aber, daß diese Hypothese besser durch eine spätere Datierung des De aedificiis gestützt wird (vgl. Signes, Procopio 67-76).

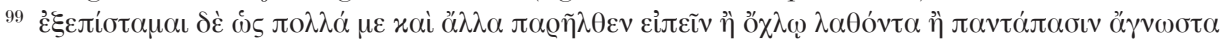

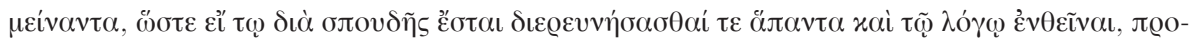

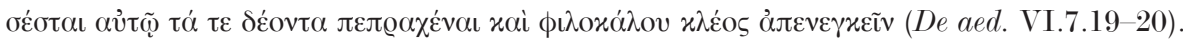


bestimmte kleine Korrekturen in seinen Büchern der Bella anbringen konn$t^{100}$. Die Leser hätten auch sicherlich nicht mehr verstanden, daß der damalige Verfasser von einem $\dot{\varepsilon} \gamma x \omega$ u einen so heftigen $\psi$ ó $\gamma$ os gegen ihn publizierte.

Wann Prokop gestorben ist, wissen wir nicht. Daß er der Präfekt sein könnte, der Belisar 562 beurteilte, ist nicht auszuschließen ${ }^{101}$, obwohl diese Möglichkeit zur Klärung des hier behandelten Problems nichts beiträgt. Keine Rolle für die Publikation der Anecdota spielt auch die Frage, ob Prokop Justinian überlebte oder nicht. Als Justinian 565 starb, war sein Neffe Justin der Nachfolger. Dieser hatte eine Nichte Theodoras, Sophia, geheiratet, die damit die neue Kaiserin wurde ${ }^{102}$. Mit Sophia rückte also wieder die Figur der bereits vor 17 Jahren gestorbenen Kaiserin in den Vordergrund. Die Publikation der Anecdota war somit für immer undenkbar geworden, obwohl der neue Kaiser Justin anfänglich von der späten Regierung Justinians (ohne Theodora!) Abstand nahm. Wie dennoch eine Kopie der Anecdota auf uns gekommen ist, läßt sich schwerlich klären. Entweder lag diese Kopie in Prokops Unterlagen und wurde nach seinem Tode entdeckt und heimlich aufbewahrt, oder es handelt sich um eine private Kopie Prokops für einen engen Freund, der den Text bei sich behielt.

\section{Anhang: Struktur und Inhalt der Anecdota}

A. Prooimion (I.1-10)

B. Belisarius und seine Frau Antonina: ihre Streitigkeiten und deren Folgen während der persischen und italienischen Feldzüge (I.11-V.27)

- I.10-14: Antonina wird als Prostituierte erzogen.

- I.15-II.14: Antoninas Untreue während ihrer Ehe mit Belisarius.

- II.15-37: persischer Feldzug des Belisarius.

- III: Kaiserin Theodora schützt Antonina und bestraft Belisarius' Sohn.

- IV: Belisarius fällt in Ungnade.

- V.1-17: Ende des zweiten italienischen Feldzuges des Belisarius.

- V.18-27: Heirat von Belisarius' Tochter.

C. Verbrechen von Sergios und Salomon in Afrika (V.28-38)

D. Justinian und Theodora während der Regierung von Justin (VI.1-X.12)

- VI.1-17: bäurische Einfalt Justins.

- VI.18-28: Verbrechen Justinians während der Regierung seines Onkels Justin (I).

- VII: Justinian und die Zirkusparteien.

100 Kislinger, Ein Angriff zu viel.

101 Signes, Procopio 16-20.

102 Cameron, The empress Sophia. 
- VIII.1-11: Verbrechen Justinians während der Regierung seines Onkels Justin (II).

- VIII.12-21: Charakterzüge Justinians (I).

- IX.1-32: die Prostituierte Theodora.

- IX.33-X.12: Justinian auf dem Sterbebett und seine Ehe mit Theodora.

E. Gesamtüberblick über die verhängnisvollen Ergebnisse der selbständigen Regierung von Justinian und Theodora (X.13-XIX.17)

- X.13-23: gemeinsame Regierung von Justinian und Theodora.

- XI.1-12: Die Gewaltherrschaft Justinians.

- XI.13-40: Ketzer und Dissidenten werden vom Kaiser verfolgt.

- XII.1-12: Beschlagnahme des Vermögens der Senatoren.

- XII.13-32: Justinian, Fürst der Dämonen.

- XIII: Charakterzüge Justinians (II).

- XIV: Korruption der Regierung.

- XV: Charakterzüge Theodoras.

- XVI.1-XVII.45: Theodoras Opfer.

- XVIII: Kriege, Katastrophen.

- XIX: Beschlagnahme des Vermögens der Untertanen.

F. Die Verwaltung des Reiches durch Justinian (XX.1-XXX.34)

- XX.1-XXI.26: Magistraturen.

- XXI.27-29: Einfälle der Barbaren.

- XXII: der Praefectus praetorio.

- XXIII: Steuerwesen.

- XXIV: Heerwessen.

- XXV: Wirtschaft.

- XXVI.1-11: städtische Verwaltung (I).

- XXVI.12-15: Konsulat.

- XXVI.16-44: städtische Verwaltung(II).

- XXVII-XXVIII: Religionspolitik.

- XXIX: Einzelfälle.

- XXX.1-16: Postverkehr und Spionage.

- XXX.17-20: Einzelfall.

- XXX.21-34: Senat. 